\title{
Non-linear processes in the interaction of atoms and molecules with intense EUV and X-ray fields from SASE free electron lasers (FELs)
}

\author{
N. Berrah ${ }^{1}$, J. Bozek ${ }^{2}$, J. T. Costello ${ }^{3 *}$, S. Düsterer ${ }^{4}$, L. Fang ${ }^{1}$, J. \\ Feldhaus $^{4}$, H. Fukuzawa ${ }^{5}$, M. Hoener ${ }^{1}$, Y. H. Jiang ${ }^{6}$, P. Johnsson ${ }^{7 \S}$, E. T. \\ Kennedy $^{3}$, M. Meyer ${ }^{8}$, R. Moshammer ${ }^{6}$, P. Radcliffe ${ }^{4}$, M. Richter ${ }^{9}$, A.

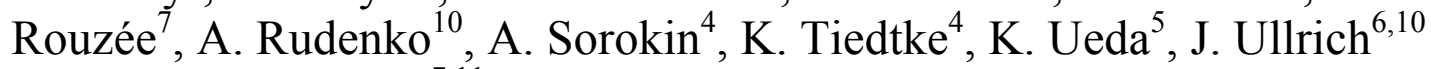 \\ and M. J. J. Vrakking ${ }^{7,11}$
}

\author{
${ }^{1}$ Physics Department, Western Michigan University, Kalamazoo, MI 49008, USA \\ ${ }^{2}$ Stanford Linear Accelerator Center, Menlo Park, CA 94025, USA \\ ${ }^{3}$ School of Physical Sciences and NCPST, Dublin City University, Dublin 9, Ireland \\ ${ }^{4}$ DESY, HASYLAB, Notkestrasse 85, D-22607 Hamburg, Germany \\ ${ }^{5}$ Institute of Multidisciplinary Research for Advanced Materials, Tohoku University, \\ Sendai 980-8577, Japan \\ ${ }^{6}$ Max-Planck-Institut für Kernphysik, Saupfercheckweg 1, 69117 Heidelberg, \\ Germany \\ ${ }^{7}$ Institute for Atomic and Molecular Physics, AMOLF, Amsterdam, The Netherlands \\ ${ }^{8}$ LIXAM/CNRS, UMR 8624 Centre Universitaire Paris-Sud, Bâtiment 350, F-91405 \\ Orsay Cedex, France \\ ${ }^{9}$ Physikalisch-Technische Bundesanstalt (PTB), D-38116 Braunschweig, Germany \\ ${ }^{10}$ Max-Planck Advanced Study Group at CFEL, 22607 Hamburg, Germany \\ ${ }^{11}$ Max Born Institute for Nonlinear Optics and Short Pulse Spectroscopy, Max-Born- \\ Straße 2 A, 12489 Berlin, Germany
}

$\S$ Now at Department of Physics, Lund University, P. O. Box 118, SE-221 00 Lund, Sweden

*Corresponding Author Email: john.costello@dcu.ie

\begin{abstract}
The advent of free electron laser (FEL) facilities capable of delivering high intensity pulses in the extreme-UV to X-ray spectral range has opened up a wide vista of opportunities to study and control light matter interactions in hitherto unexplored parameter regimes. In particular current short wavelength FELs can uniquely drive nonlinear processes mediated by inner shell electrons and in fields where the photon energy can be as high as $10 \mathrm{keV}$ and so the corresponding optical period reaches below one attosecond. Combined with ultrafast optical lasers, or simply employing wavefront division, pump probe experiments can be performed with femtosecond time resolution. As single photon ionization of atoms and molecules is by now very well understood, they provide the ideal targets for early experiments by which not only can FELs be characterised and benchmarked but also the natural departure point in the hunt for nonlinear behaviour of atomistic systems bathed in laser fields of ultrahigh photon energy. In this topical review we illustrate with specific examples the gamut of apposite experiments in atomic, molecular physics currently underway at the SCSS test accelerator (Japan), FLASH (Hamburg) and LCLS (Stanford).
\end{abstract}

Keywords: free electron laser; FLASH; LCLS; SCSS Test Accelerator; extreme-ultraviolet; Xray; atom; molecule; multiphoton; ionization; dissociation; photoelectron; photoion; pumpprobe 


\section{Introduction}

The development of extreme-UV and X-ray lasers of high peak and average power eluded physicists and engineers until the successful realisation of 'Self Amplified Spontaneous Emission' or SASE in a free electron laser (FEL) at the Tesla Test Facility in DESY on February $22^{\text {nd }}, 2000$ at the relatively high photon energy of 11.5 $\mathrm{eV}$ [1]. In mid 2005 the FEL at DESY, now known as 'FLASH' or Free electron LASer in Hamburg [2] opened as a user facility offering EUV radiation in the 20 $200 \mathrm{eV}$ photon energy range in pulses of duration $10-30 \mathrm{fs}$ carrying an average energy of $50 \mu \mathrm{J}$ per pulse [3]. Since then two new short wavelength FELs have begun operation. In 2007 the Spring-8 Compact SASE Source (SCSS) test accelerator in Japan [4], opened at softer photon energies than FLASH (typically $20-24 \mathrm{eV}$ ) and the Linac Coherent Light Source (LCLS) in the USA [5] which operates at harder photon energies in the X-ray region, currently $800-2000 \mathrm{eV}$, for atomic and molecular physics experiments, opened to users in mid 2009. Like FLASH these sources provide typically $10^{13}$ photons in pulses of $\sim 100$ fs (SCSS test facility) and 100 - 250 fs (LCLS) duration. The latter FEL has a so-called low current mode of operation that provides pulses with a duration of $1-4$ fs. The interested reader can find comprehensive information on the underlying theory, design and operation of short wavelength FELs in a number of reviews and overviews $[6,7,8,9,10]$ while descriptions of the broader range of experiments possible in AMO physics, condensed matter, imaging etc. carried out at FLASH can be found in references [11, 12].

The focus of this particular review is on experiments designed to observe nonlinear processes induced by the intense high frequency driving field, a unique selling point of EUV and X-ray FELs and to track the time evolution of ionization and 
dissociation on ultrashort timescales. We will do this by describing a selection of recent experiments carried out at the SCSS test accelerator, FLASH and LCLS. We will confine ourselves to gas phase atomic and molecular targets since they have been well characterised already in weak field EUV/ X-ray photoionization studies while their behaviour in intense optical laser fields is becoming well established.

Since it is instructive to outline some of the gross features of the interaction of intense laser fields with atoms (or molecules) and indicate how these are modified when the photon energy is ramped up into the EUV and X-ray ranges we will end this section with a short discussion on this topic. The study of atoms and molecules in intense infrared and visible laser fields has revealed many intriguing dynamical processes such as multi-photon, above-threshold and optical-field ionization [13] as well as the production of short wavelength coherent radiation via high harmonic generation yielding attosecond pulses [14]. Ionization pathways for atoms and molecules in laser fields depend on a combination of ionization potential, laser photon energy and laser intensity. Theoretical descriptions can be made within a perturbative framework or may require a full solution of the time dependent Schrödinger equation depending whether the process resides within the multiphoton (MPI), tunnel (TI) or the related over the barrier (OBI) ionization regimes. A simple but useful and instructive parameter $(\gamma)$ that determines in which regime a particular experiment is likely to lie is due to Keldysh [15]:

$$
\gamma=\sqrt{\frac{I P}{2 U_{p}}} \text { where } U_{P}=9.3 \times 10^{-14} I\left(W_{c m}^{-2}\right) \lambda^{2}(\mu m) e V
$$

Here IP is the ionization potential and $\mathrm{U}_{\mathrm{p}}$ is the so-called ponderomotive potential (the cycle averaged kinetic energy of a liberated electron in the laser field) while I and $\lambda$ are the laser power density and wavelength respectively. For $\gamma>>1$ the interaction is definitively as discretized multiphoton ionization while for $\gamma<<1$ it is best described 
in field ionization terms As the laser photon energy is tuned to the EUV, the ponderomotive potential drops quadratically and the Keldysh parameter increases linearly. Hence EUV FELs will tend to favour multiphoton effects that are readily described by pertubation methods.

One way to view this result is as follows; when the photon energy lies well above the ionization potential, the tunnel ionization rate will be less than the laser frequency and so ionization via single and multiple photon absorption are the more favoured routes. From another viewpoint, as the laser photon energy increases, fewer photons are needed to ionize the atoms and so the ionization probability becomes quite high at relatively low intensity. Taking the case of $\mathrm{He}$, for a laser intensity of $10^{16} \mathrm{~W} / \mathrm{cm}^{2}, \gamma \sim 0.05$ (tunnel ionization regime) for a Ti-Sapphire laser emitting 800 $\mathrm{nm}(\mathrm{h} v \sim 1.5 \mathrm{eV})$ radiation, while for a FEL operating at $8 \mathrm{~nm}(\mathrm{~h} v \sim 150 \mathrm{eV}), \gamma \sim 4.5$ (the MPI regime). The difference is highly significant since it means that EUV and Xray FELs reassert the primacy of the photon in high intensity laser-matter interactions and enter into a domain where few photon processes reign and perturbative methods can be used to describe them quite accurately.

As one might expect, there has been a surge in theoretical activity on this topic since the late nineties when firm design parameters for short wavelength FELs began to appear in the literature [16]. A pioneering (and indeed prescient) paper on intense EUV-atom interactions due to Lambropoulos and Zoller appeared as early as 1981 [17]. It is not the objective of this paper to review the large body of relevant theoretical work, already numbering in the hundreds. For the interested readers the recent papers on double ionization in intense EUV fields by Lambropoulos et al. [18] and Fritzsche et al [19] represent a suitable starting point for a trip through the recent history of relevant theoretical activity. 
The paper is organised as follows. In the next section we describe the range of experiments that have observed non-linear behaviour in atoms and molecules in intense monochromatic and polychromatic fields at the Japanese EUV FEL, FLASH and LCLS. Examples will include, simultaneous and sequential multiphoton and/or multielectron ionization of atoms, molecular ionization and dissociation in intense fields extending from the VUV to the X-rays, ionization processes in superposed EUV and IR fields, time resolved dissociation and ionization of molecular targets in EUV pump-EUV probe experiments and evolution of dynamically aligned molecules in two colour (EUV + IR) fields (including photodissociation with a third UV laser). We end the article with a summary of work currently in progress and planned for the near future.

\section{Recent results from experiments at the SCSS test accelerator, FLASH and LCLS - atoms and molecules in strong EUV and X-ray laser fields.}

We present results from each these facilities in the natural order of SCSS test accelerator (EUV), FLASH (EUV) and LCLS (X-ray). As LCLS only began user operation in mid 2009, most of the data obtained at that facility to date is under analysis. Hence we focus the discussion on its dedicated AMO physics end-station and show just one illustrative, first result on molecular dissocation/ ionization in an Xray field.

\section{II.1 Experiments at the SCSS Test Accelerator}

\section{II.1.1 AMO science at SCSS test accelerator in Japan (Sendai-Tohoku University)}

As mention in introduction, the SCSS test accelerator in Japan $[4,20]$ provides linearly polarized EUV-FEL radiation $(\sim 30 \mu \mathrm{J}$ per pulse, $\sim 100 \mathrm{fs}$ pulse width, $10-20$ $\mathrm{Hz}$ repetition rate) in the wavelength region 51-62 $\mathrm{nm}$ at saturation. This energy 
regime is of particular interest because all atoms, except helium, in any forms of matter, can be ionized with huge photoionization cross sections.

This new source has been in operation for user experiments since May 2008. It, however, required us users to redesign the experimental end stations and the particle detection systems compared to those used at synchrotron sources or optical laser sources with high repetition rates $\geq 1 \mathrm{kHz}$. For example, the use of delay line detectors has become a widespread method for multi-particle momentum spectroscopy both for synchrotron radiation experiments [21] and laboratory laser experiments with high repetition rates (1 kHz or higher) [22]. This well-established approach is, however, limited to detecting a few particles from a single event, or from a single light pulse. For the shortwavelength FELs, a single pulse produces hundreds of ions from the target and residual gases. Thus, the appropriate data acquisition system must be capable of recording several tens of ions shot by shot, assuming the ion detection efficiency to be $10 \%$. Though higher values of the overall detection efficiency are desirable in most cases, the danger of systematic errors due to saturating the detection system is the main concern for FEL experiments.

To circumvent difficulties described above, a dead-time free ion detection system has been developed within the framework of international collaborations [23] and have been investigating multiple ionization of atoms [24] and molecules [25-30]. In what follows we describe the design of the apparatus, the dead-time free ion detection system, and the experimental alignment, we illustrate experimental results and discuss sequential multiple ionization of atoms and molecules and conclude with a summary and outlook.

The design of our ion spectrometer is shown in figure 1 . It is very similar to the ones described in Refs. [31,32]. The spectrometer uses two acceleration regions 
with homogenous electric fields and a $308 \mathrm{~mm}$ long drift tube. The source volume of the ions is the intersection of the gas beam with the FEL focus located $40 \mathrm{~mm}$ away from the extractor electrode and $35 \mathrm{~mm}$ away from the pusher electrode. The pusher and extractor electrodes are plane aluminium sheets with round holes that are covered by flat stainless steel meshes. The second acceleration region is $51 \mathrm{~mm}$ long. After the second acceleration region the extracted ions enter the $308 \mathrm{~mm}$ long drift tube terminated by two grids to obtain a field free region internally. The voltages on the electrodes are usually set to values that fulfil the space focusing condition. Using 128 $\mathrm{V} / \mathrm{mm}$ and $226 \mathrm{~V} / \mathrm{mm}$ in the two acceleration stages, respectively, we can project all ions with kinetic energies up to $20 \mathrm{eV}$ onto a $120 \mathrm{~mm}$ diameter microchannel plate (MCP) in front of the delay line detector. The determination of the ion momentum is based on the measurement of the time-of-flight (TOF) and the detector hit position for each individual ion. See, for example, [21, 33] for more general information about this technique. Here, a three-layer HEX type detector is used to minimize the deadtime [34].

For typical experimental conditions, many singly charged ions are released in a single FEL shot and the TOF and detector hit positions of approximately $10 \%$ (determined by the detection efficiency) must be recorded within a short time window of some $100 \mathrm{~ns}$. Depending on the hit position the time that a pulse needs to leave the delay line anode is $0-100 \mathrm{~ns}$, therefore a substantial amount of temporal overlap of the signals from different ions occurs. A combination of the redundant information from a three layer delay line detector and a sophisticated logic is necessary to reconstruct all hit positions and momenta. Instead of conventional constant-fraction discriminators and time-to-digital converters, an 8-channel digitizer (Acqiris DC282× 2 ) is used $[35,36]$. The trigger signal is derived from the master oscillator of the 
FEL. The complete waveforms of six signals from the three-layer delay-line anode and one from the MCP are recorded by seven channels of the 8-channel digitizer and stored in the computer. The timing signals are extracted off line from each waveform by software resembling a constant-fraction discriminator [37]. We restrict the analysis to ions whose signal pulses do not overlap on at least two layers. The detected position and TOF of each ion are obtained from the seven different timing signals and then the $3 \mathrm{D}$ momentum is calculated using the position and TOF information for the individual ions. The redundancy of the data set (only 4 out of 7 readouts are essential) allows us to perform a virtually dead-time-free measurement for the 3D momenta of up to 100 ions produced by a single shot.

The schematic experimental alignment is shown in figure 2. The time-of-flight axis of the spectrometer is vertical and thus perpendicular to the horizontal axes of the FEL beam and of its polarization. The supersonic molecular beam is horizontal and crosses the FEL beam at 45 degrees. The FEL beam is steered by two upstream Au or SiC plane mirrors, skimmed by a $7 \mathrm{~mm}$ hole $1.2 \mathrm{~m}$ upstream from the entrance of the experimental chamber, and then introduced to the chamber placed $\sim 26 \mathrm{~m}$ downstream from the radiation source point. To focus the FEL beam onto the molecular beam of $\sim 1 \mathrm{~mm}$ in diameter, we use a $\mathrm{Mg} / \mathrm{Si}$ multilayer concave mirror with a focal length of $250 \mathrm{~mm}$ at normal incidence [24]. The diffraction-limited focal spot size is estimated to be $\sim 3 \mu \mathrm{m}$ and the reflectivity to be $20-40 \%$ at $51-62 \mathrm{~nm}$. The FEL beam was partially blocked by a $1.5 \sim \mathrm{mm}$ wide horizontal beam stopper (see the inset of figure 2) so that the non-focused beam did not irradiate directly the molecular beam. The intensity of the FEL beam is adjusted using a gas attenuator, i.e., an absorption cell filled with the Ar gas. Taking all the optical elements (steering and focusing mirrors, skimmers, etc) between the radiation point and the ionization point into account, we 
expect the power density at the focus spot on the atomic beam at most $1-2 \times 10^{14}$ $\mathrm{W} / \mathrm{cm}^{2}$ at full power of the FEL $(30 \mu \mathrm{J})$. We consider this number as an upper limit because it relies on the designed values of the focusing mirror given above and thus we expect that the uncertainty may be a factor of 4 mostly due to the uncertainty of the spot size.

We have explored multiple ionization of $\mathrm{Ne}, \mathrm{Ar}, \mathrm{Kr}$ and $\mathrm{Xe}$ by EUV FEL pulses at 51 and $62 \mathrm{~nm}$, varying the FEL intensity. Preliminary results for Ar irradiated by $62 \mathrm{~nm}$ FEL pulses were reported in [24]. Figure 3 shows TOF spectra of Ar and $\mathrm{Kr}$ irradiated by $51 \mathrm{~nm}(\mathrm{~h} v \sim 24 \mathrm{eV})$ FEL pulses. One can see highly charged ions up to $7+$ for $\mathrm{Ar}$ and $8+$ for $\mathrm{Kr}$. The total energies required to remove seven electrons from the Ar atom and eight electrons from the $\mathrm{Kr}$ atom, producing $\mathrm{Ar}^{7+}$ and $\mathrm{Kr}^{8+}$, respectively, are $\sim 434$ and $\sim 508 \mathrm{eV}$, i.e., more than 18 and 21 times the photon energy of $24 \mathrm{eV}$. Here the question is whether the electron strip is simultaneous or sequential. If multiple ionization is due to the sequential stripping of outermost electrons, then the total numbers of photons absorbed by a single atomic $\mathrm{Ar}$ and $\mathrm{Kr}$ should be 22 and 26 , respectively.

Figure 4 depicts the FEL power dependence, $p_{\mathrm{A}}$ of the ion yields for different charge states of Ar irradiated by $62 \mathrm{~nm}(\mathrm{~h} v \sim 21 \mathrm{eV})$ FEL pulses [24], where I is the FEL intensity. $\mathrm{I}=1$ here corresponds to the average value for the full FEL flux. Employing the estimates of the maximum possible power density given in the previous section, $\mathrm{I}=1$ corresponds to a power density of $2 \times 10^{14} \mathrm{~W} \mathrm{~cm}^{-2}$. Unlike the absolute power density, the relative values of the power densities are quite accurate. Values of the slope $p_{\mathrm{A}}$ for $\mathrm{Ar}^{n+}$ yields, obtained by fitting to the data points below the saturation, are also given in figure 4. Looking at values of the differences $\Delta p_{\mathrm{A}}$ in the slope of the charge states $n+1$ and $n$, we find the following: 
1) $\mathrm{The}^{2+}$ yield depends quadratically on $I$ below the saturation point. This quadratic dependence correlates well to the number of photons necessary to remove one more electron from $\mathrm{Ar}^{+}$whose yield has already been completely saturated. 2) The difference $\Delta p_{\mathrm{A}}$ in slopes $p_{\mathrm{A}}$ between the $\mathrm{Ar}^{2+}$ and $\mathrm{Ar}^{3+}$ yields below the saturation is 2.6. To ionize the $\mathrm{Ar}^{2+} 3 \mathrm{p}^{-2}{ }^{3} \mathrm{P}$ ground triplet states, three photons are required. To ionize the $\mathrm{Ar}^{2+} 3 \mathrm{p}^{-2}{ }^{1} \mathrm{D}$ singlet excited state, on the other hand, two photons are enough. Thus, the difference $\Delta p_{\mathrm{A}}=2.6$ in slopes is consistent with subsequent removal of one electron from $\mathrm{Ar}^{2+}$.

3) The difference $\Delta p_{\mathrm{A}}=3.1$ in slopes between $\mathrm{Ar}^{3+}$ and $\mathrm{Ar}^{4+}$ is also in reasonable agreement with the number of photons 3 required to remove one electron from $\mathrm{Ar}^{3+}$.

These observations indicate that the multiple ionization proceeds via the sequential stripping of electrons. The large uncertainties in the slope, however, start to mask a decisive conclusion for the highly charged states 4+ and higher. Analyses of other rare gas atoms at other photon energies are in progress together with relevant theoretical studies.

Figure 5 shows the photoion-photoion coincidence (PIPICO) map for $\mathrm{N}_{2}$ irradiated by $51 \mathrm{~nm}(\mathrm{~h} v \sim 24 \mathrm{eV})$ FEL pulses. In this figure, ion-pairs satisfying the momentum conservation law only for a plane perpendicular to the time-of-flight axis are shown. One can clearly see the correlation lines due to $\mathrm{N}^{+}-\mathrm{N}^{+}, \mathrm{N}^{+}-\mathrm{N}^{2+}, \mathrm{N}^{2+}-\mathrm{N}^{2+}$, and $\mathrm{N}^{2+}-\mathrm{N}^{3+}$ from $\mathrm{N}_{2}$, even though the number of molecules at the FEL focus point is much more than one. Relying on the momentum conservation law, we can extract the two-body breakup channels of the $\mathrm{N}_{2}$ molecule ionized by FEL to charge state up to $5+$.

The observation can be straightforwardly extended to three-body breakup of polyatomic molecules. Figure 6 shows a PIPIPICO map for $\mathrm{C}^{2+}-\mathrm{O}^{2+}-\mathrm{O}^{2+}\left(\right.$ and $\mathrm{C}^{+}-\mathrm{O}^{2+}-$ 
$\mathrm{O}^{2+}$ ) coincidences from $\mathrm{CO}_{2}$ irradiated by $62-\mathrm{nm}$ EUV-FEL. In this figure, three ions satisfying the momentum conservation law for a plane perpendicular to the time-offlight axis are shown. We can extract three-body breakup channels of $\mathrm{CO}_{2}$ ionized by FEL to charge state up to $6+$.

Figure 7 shows the kinetic energy release (KER) distribution for each of the ion-pair formation channels of $\mathrm{N}_{2}$ found in figure 5 [26]. The horizontal axis indicates the KER divided by Q, where $\mathrm{Q}$ is the product of the charges of both ions. For the spectrometer setting in this work, we can collect all singly, doubly and triply charged ions emitted into $4 \pi$ sr with kinetic energies up to $\sim 20, \sim 40$ and $\sim 60 \mathrm{eV}$, respectively. We can see that KER/Q decreases with the increase in the total charge of both ions. An axis on top of the figure indicates the internuclear distance estimated by the Coulomb repulsion energy. For $\mathrm{N}^{2+}-\mathrm{N}^{2+}$ and $\mathrm{N}^{2+}-\mathrm{N}^{3+}$ ion-pair formations, the KER distributions are peaked at values significantly below the Coulomb repulsion energy at $\mathrm{R}_{0}$. These observations indicate that the internuclear distance is elongated during the multiple ionization processes. If multiple-ionization was caused by "direct" vertical multi-photon absorption processes, the internuclear distance would be equal to $\mathrm{R}_{0}$, leading to $\mathrm{KER} / \mathrm{Q}$ as high as $13 \mathrm{eV}$, certainly not found in our result. Thus, the observed KER distributions clearly indicate that multiple ionization occurs via step-wise electron removal proceeding sequentially within the $100 \mathrm{fs}$ pulse duration, i.e. via sequential multi-photon absorption. We find similar results also for $\mathrm{O}_{2}$ and $\mathrm{CO}_{2}$.

In summary we have developed a dead-time free ion detection system and used it to investigate multiple ionization of atoms, molecules, and clusters by EUV FEL pulses at the SCSS test accelerator in Japan. For the atoms and molecules concerned here, we have confirmed that multiple ionization proceeds via sequential 
stripping of electrons by FEL pulses with wavelengths in the range 51-62 nm and for power densities $<10^{14} \mathrm{~W} \mathrm{~cm}^{-2}$. In order to investigate further details of the interaction of EUV FEL pulses with atoms, molecules, and clusters, however, electron spectroscopy is indispensable and is in progress. Another important subject of AMO science with the SCSS test accelerator, besides the non-linear spectroscopy discussed here, is pump-probe experiments. Both EUV-pump-EUV-probe and IR/UV-pumpEUV-probe experiments are in preparation, aiming to explore time-resolved electron and nuclear dynamics in photo-excited molecules and clusters.

\section{II.2 Recent experiments at FLASH}

FLASH has been in operation since mid 2005 and so has hosted the largest range of experiments of the currently three operational light sources There are now in excess of 100 papers in the open literature on results from FLASH with atomic and molecular targets. We take here a selection of very recent illustrative results. The beamlines and diagnostics at FLASH have been described in detail recently by Tiedtke et al. [38].

II.2.1 Non-linear Photoionization in the Soft X-ray Regime (Berlin-PTB, St. Petersburg-Ioffe Institute and Hamburg-DESY)

In two recent rare gas photoionization studies $[39,40]$ applying ion spectroscopy at FLASH in the extreme ultraviolet (EUV), exceptional behavior of light-matter interaction at high irradiance has been demonstrated: At a few $10^{15} \mathrm{~W} \mathrm{~cm}^{-2}$, nearly 50 EUV photons of $90 \mathrm{eV}$ photon energy interact within 10 to 20 femtoseconds with a single xenon atom and lead to the emission of up to 21 electrons. Neon, argon, and krypton do not show this high degree of photoionization under the same experimental 
conditions. A controversial discussion has started on the interpretation of these results and on the role of the so-called $4 \mathrm{~d}$ giant resonance in this context [41]. This resonance is, in comparison to the other rare gases, a particular feature of xenon in the EUV and arises in the photon energy range from about 85 to $115 \mathrm{eV}$ [42]. For many years, it represents a prime example for the impact of electron correlation on inner-shell photoionization at low irradiance [43]. The proposal [39] that the strength and nature of this resonance may also drive, at high irradiance, direct multiphoton excitation from the inner $4 \mathrm{~d}$ shell constitutes the core of the controversy.

The experiments were carried out by applying ion Time-Of-Flight (TOF) mass/charge spectroscopy in the 3 to $5 \mu \mathrm{m}$ focus of a spherical multilayer mirror. Irradiance levels up to $10^{16} \mathrm{~W} \mathrm{~cm}^{-2}$ in the Extreme Ultra-Violet (EUV) were achieved. Figure 8 shows the result for Xe obtained at $93 \mathrm{eV}$ photon energy. It refers to the nonlinear appearance of higher charge states with increasing irradiance $E$. At $E=7.8 \times$ $10^{15} \mathrm{~W} \mathrm{~cm}^{-2}, \mathrm{Xe}^{21+}$ occurs whose generation, starting from neutral Xe, requires a total energy of at least $5 \mathrm{keV}$. Plasma effects like electron impact from neighboring atoms can be excluded because the spectra do not depend on the target density. It means that almost 60 EUV photons at the energy of $\hbar \omega=93 \mathrm{eV}$ must have been absorbed by a single atom within the estimated FLASH pulse duration of 10 to 20 fs. A satisfying $a b$ initio calculation of these findings within the framework of high-order perturbation theory and individually interacting photons seems difficult to perform. On the other hand, semi-classical models within the wave picture of light, as developed for strongfield phenomena in the optical regime $[13,15,44]$, also seem to fail. For their application, the ponderomotive energy defined in Eq. 1 which is the quiver energy transferred to a free electron by the oscillating field, is far too low $(<0.2 \mathrm{eV})$. 
The xenon results are in clear contrast to former works on non-linear gasphase photoionization performed at FLASH. The ion, electron, and reaction microscope spectra of molecular nitrogen [45], argon [46], neon [46, 47], and helium $[47,48]$ can generally be well interpreted in terms of target depletion, space-charge effects, and sequential and direct two-, three-, and four-photon processes as well. In contrast to xenon [39], here the non-linear dependence of the photoionization rate of an $\mathrm{m}(>1)$-photon process $\dot{N}_{p}^{(\mathrm{m})}$ on irradiance $E$ derived from $\mathrm{m}^{\text {th }}$-order perturbation theory is largely confirmed:

$$
\dot{N}_{p}^{(\mathrm{m})}=N \sigma^{(\mathrm{m})}\left(\frac{E}{\hbar \omega}\right)^{\mathrm{m}}
$$

$N$ denotes the target number within the interaction volume and $\sigma^{(\mathrm{m})}$ the generalized cross section for the m-photon process. However, even for one of the simplest showcases in this context, i.e. the direct two-photon double ionization of helium, a satisfying theoretical prediction from first principles still represents a challenge. Calculations of the corresponding generalized cross section differ by almost one order of magnitude as demonstrated by figure 9 [47, 49-56]. The first experimental data, estimated from a High-Harmonic Generation (HHG) experiment performed at 41.8 $\mathrm{eV}[49,50]$ and resulting from a more quantitative study performed at $42.8 \mathrm{eV}$ at FLASH [47], lie well in between the two main groups of theoretical values [51-56] (figure 9). Good agreement with the experimental results is achieved, so far, in the recent work by Ivanov and Kheifets [55].

In conclusion, non-linear photoionization in the soft X-ray regime represents still a largely unexplored territory. Further experimental and theoretical efforts seem to be necessary to understand the mechanisms of photon-matter interaction at high photon intensity in conjunction with high photon energy. The application of electron 
and fluorescence spectroscopy at the upcoming soft X-ray free-electron laser facilities will certainly make a major contribution in this context.

II.2.2 Few-photon - few-electron interactions in atoms and molecules (HamburgDESY-CFEL and Heidelberg-MPIK)

As described earlier, Free Electron Lasers (FEL) like the FLASH at Hamburg [12], the SCSS test facility in Japan [4] or the LCLS at Stanford [5, 57], deliver light pulses from the extreme ultra-violet (EUV) to X-ray energies with unprecedented intensity $\left(10^{15} \mathrm{~W} / \mathrm{cm}^{2}\right.$ and more are routinely achieved) and ultra-short durations in the $100 \mathrm{fs}$ regime even reaching less than 7 fs [57]. Among others, they enable for the first time differential measurements on basic non-linear process where few EUV photons interact with few electrons leading to double or multiple ionization of atoms and molecules. Experimental studies of such interactions are not only of practical relevance for research at FELs in general, since such processes essentially underlie all radiation-matter interactions in any experiment at typical FEL intensities, but they are of utmost scientific importance to guide and to verify basic few-electron quantum theories. Beyond such detailed studies of purely non-linear processes a whole class of new experiments is emerging exploiting the short pulse duration in combination with advanced pump-probe schemes to trace in real-time and with fs resolution the dynamical evolution of excited atoms, molecules or clusters with EUV photons. First results of EUV-pump - EUV-probe experiments with molecules as well as differential data for two-photon double ionization (TPDI) will be presented in this section. We briefly describe the experimental method before discussing recent results on (i) direct and sequential TPDI of $\mathrm{He}$ and $\mathrm{Ne}$, (ii) two-photon induced Coulomb explosion of $\mathrm{D}_{2}$, as well as (iii) time-resolved fragmentation of highly excited $\mathrm{N}_{2}$ molecules. 
In several experimental campaigns at FLASH the Heidelberg reaction microscope (REMI), a dedicated multi-particle imaging spectrometer which allows measuring the three-dimensional (3D) momentum vectors of all charged fragments, ions and electrons, was used to explore non-linear reactions in atoms and small molecules. The FEL beam (typically 30 micro-bunches at $5 \mathrm{~Hz}$ repetition rate) was focused onto a collimated supersonic gas-jet target $\left(10^{10}\right.$ particles $\left./ \mathrm{cm}^{2}\right)$ in the ultrahigh vacuum chamber (10 $\left.{ }^{-11} \mathrm{mbar}\right)$ of the REMI (for details see Ref. [33]). By means of weak electric $(1-50 \mathrm{~V} / \mathrm{cm})$ and magnetic $(10 \mathrm{G})$ fields applied along the vertical axis (horizontal laser polarization) the created ions and electrons are guided onto two position-sensitive and multi-hit capable MCP detectors (diameter $120 \mathrm{~mm}$, position resolution $0.1 \mathrm{~mm}$, dead-time $10 \mathrm{~ns}$ ) achieving a detection solid-angle close to $4 \pi$ for all particles of interest. From the measured TOF and position on the detector the initial momentum vectors of emerging ions and electrons were reconstructed.

Even though considerable progress has been achieved (see e.g. [58 - 61] and references therein) as a result of impressive efforts from several theory groups during the last few years, the most basic and, therefore, most fundamental non-linear reaction, direct two-photon double ionization of $\mathrm{He}$, is still a subject of controversial debate. Total cross section predictions differ by up to a factor of 20 (see Figure 9) and the situation on the experimental side as well, with only two data points for $\mathrm{He}$ measured with high harmonic radiation [49] and at FLASH [47], is far from satisfactory (cf: figure 9), due to the essentially unknown pulse duration (at FLASH) and limited statistics, the experimental results exhibit relatively large error bars such that they can hardly discriminate between different theoretical predictions. As already discussed in the previous section, the situation becomes even more confusing at higher intensities and for more complex atoms like Xe, where charge states up to $\mathrm{q}=$ 
21 have been observed recently [39] and are subject of intense discussion in the literature (see e.g. [41]).

The REMI can provide differential information. Figure 10(a) shows the momentum distribution of recoiling $\mathrm{He}^{2+}$ ions measured at $44 \mathrm{eV}$ [62]. At this energy two-photon double ionization is only possible by a direct transition from the ground to the doubly ionized state, all sequential pathways via intermediate $\mathrm{He}^{+}$states are energetically forbidden. The circular-shaped pattern with its maximum at zero momentum indicates that both electrons are preferentially emitted with similar energies into opposite hemispheres, leaving the momentum balancing nucleus almost at rest. This is quite different to one-photon double ionization, where parity and angular momentum selection rules prohibit a back-to-back emission of equal energy electrons. Despite the low statistics these first differential data on TPDI of He already serve as a test of theory and have triggered a wealth of theoretical work with partly conflicting results as well [58 - 61]. More recently, we have taken data at $52 \mathrm{eV}$ with high statistical significance that helped uncover deficiencies of earlier calculations [63].

The situation changes when atoms with lower ionization potentials or higher photon energies are used such that a step-wise or sequential ionization via real intermediate ionic states becomes possible as for double ionization of $\mathrm{Ne}$ at $44 \mathrm{eV}$ shown in figure 10(b) [46]. Now, the momentum distribution exhibits a dipolarshaped momentum distribution reflecting the emission of the $22 \mathrm{eV}$ photo-electron in the first step whereas the subsequently emitted low-energy electron from $\mathrm{Ne}^{+}$leads to a broadening of the ring-like momentum pattern only. In contrast to the general opinion that sequential ionization is less interesting than direct TPDI, because it is often considered as consisting of two independent ionization steps, recent calculations 
[19] along with first kinematically complete REMI measurements [64] showed that both electrons are correlated, even entangled. Future time-resolved experiments using e.g. pump-probe setups as those described below will certainly reveal further surprises advancing our understanding of this important non-linear reaction channel not only in atoms, but also in molecules. In general, for not too low photon energies, sequential ionization is the dominant process in more complex molecules due to the large number of intermediate states that are available. A detailed study on multiple ionization pathways inspecting the angular and energy dependent emission of ionic fragments for different charge states after fragmentation of $\mathrm{N}_{2}$ has revealed a wealth of interesting features [65].

Whenever the sequential ionization becomes energetically possible the question arises about the relative importance of the two TPDI pathways, in particular if molecules are exposed to intense EUV radiation. In a recent experiment at FLASH in a collaborative effort with theory we were able to disentangle both channels, sequential and direct TPDI, for Coulomb explosion of $\mathrm{D}_{2}$ at $38 \mathrm{eV}$ by inspecting the coincident $\mathrm{D}^{+}$fragment kinetic energies [66]. The resulting kinetic energy release (KER) spectrum (figure 11(a)) reveals two maxima where the high energy part at 18 $\mathrm{eV}$ corresponds to a Franck-Condon transition from the molecular ground-state to the repulsive 1/R Coulomb state (R: inter nuclear distance). Direct TPDI will lead to such high KER, but in addition sequential ionization with a very short time gap (much less than the Coulomb explosion time) between both transitions may also contribute. The low KER region, however, is accessible via sequential ionization only enabling a quantitative separation of both pathways with the help of theory. Advanced manyparticle quantum calculations by F. Martin et al. [66] deliver absolute cross sections for the sequential part along with relative KER distributions for direct TPDI such that, 
in comparison with experiment, not only both pathways can be disentangled but, moreover, first conclusions on the relative importance of both processes can be obtained. Moreover, even the fragment ion angular distribution (figure 11(b)) can be extracted and is found to be in good agreement with the experimental results as well. Certainly, time-resolved (pump-probe) experiments will enable a much deeper insight into the combined nuclear and electronic dynamics. Just recently we succeeded in performing such a measurement where the motion of the intermediate $\mathrm{D}_{2}{ }^{+}$nuclear wave-packet (vibrational period $22 \mathrm{fs}$ ) was followed in real-time between the two ionization events from zero up to $100 \mathrm{fs}$ (see upcoming publication [67]).

In order to perform EUV pump-probe experiments our reaction microscope was equipped with a back-reflecting split-mirror setup for the generation of two FEL pulses that are time delayed with respect to each other. Pump and probe pulses are thus identical apart from their relative intensity that can be chosen at will by varying the relative illumination of both parts by the incoming light. The set-up consists of a spherical multi-layer mirror (30-60 cm focal length) that is cut into two identical "half-mirrors". While one half is mounted at a fixed position, the other one is movable by means of a high precision piezo-stage along the beam axis (delay range $\pm 1500 \mathrm{fs}$, resolution $1 \mathrm{fs}$ ). The foci of both mirrors are merged inside the centre (gas-jet) of the REMI via tip and tilt motions of one half-mirror.

For a prototype EUV pump-probe experiment $\mathrm{N}_{2}$ was chosen as target because it has been investigated in great detail theoretically as well as experimentally including recent VUV-attosecond pump-probe measurements providing rich information on the fragmentation dynamics of excited ionic states [68]. With intense FEL radiation the molecule gets singly or even multiply ionized by the first pump pulse while the dissociating or Coulomb exploding molecule is probed by further 
ionization in the second pulse. The kinetic energy of created $\mathrm{N}^{2+}$ ions emerging from up to 6-fold ionized molecules is plotted in figure 12 as a function of the pump-probe time delay (photon energy $38 \mathrm{eV}$ ). With increasing time the fragment energy decreases. This can possibly be explained by a scenario where first a highly excited most likely doubly charged molecular ion is created that starts to dissociate (there are e.g. plenty of metastable states of the dication $\mathrm{N}_{2}{ }^{2+}$ ) and gets further ionized by the delayed probe-pulse during its course of fragmentation. The growing inter-nuclear distance with time leads to smaller and smaller Coulomb-explosion energies. We observe pronounced and strikingly different time dependences for different ionic states, determined by the coincident charged fragment detection and their KERs. Though at the present stage of ongoing data analysis these states are not yet fully identified, we are convinced that this experiment sets the starting point for a large variety of time-resolved future investigations. Among many others, detailed studies of dynamically evolving highly excited molecular states, of light-induced conformational changes in molecules (isomerization) or investigations of inherently difficult to describe couplings between electronic and nuclear degrees of motion in molecules (e.g. at conical intersections) will become possible using intense fs FEL pulses in pump-probe experiments.

\section{II.2.3 Ionization in intense EUV and IR laser fields (Dublin-DCU, Hamburg-DESY and Orsay-LIXAM)}

The unprecedented characteristics of FLASH open up new research opportunities also for two-colour studies of atomic photoionization. For example, non-linear processes involving innershell electrons can be studied by combining and synchronizing the FEL pulses with those of an intense ultrashort pulse laser [69]. Due to the short pulse 
width of the FEL, the study of multi-photon processes induced by the strong optical field are possible. Alternatively the high intensity of the FEL opens up also twophoton processes, either in a two-colour arrangement combining FEL and optical pulses or by inducing one-colour non-linear processes by the FEL only.

The experiments were performed using the two-colour pump-probe set-up at FLASH [70]. Briefly, the EUV FEL and the optical laser beams were introduced into the vacuum chamber in a collinear geometry and intersect an effusive gas jet within the acceptance volume of a magnetic bottle electron spectrometer (MBES). The high collection efficiency of the MBES made it possible to record energy-resolved electron spectra for each individual FEL pulse. The FEL was operated in single-bunch mode at a $5 \mathrm{~Hz}$ repetition rate and fundamental wavelength around $13.7 \mathrm{~nm}$ with a mean pulse energy of about $20 \mu \mathrm{J}$. The optical field was produced by Ti:sapphire laser systems delivering either ultra-short optical pulses (120 fs, $800 \mathrm{~nm}, 20 \mu \mathrm{J})$ at the $\mathrm{MHz}$ repetition rate of the multi-bunch mode of FLASH or pulses of variable pulse duration and energy (up to $2 \mathrm{~mJ}, 800 \mathrm{~nm}, 120 \mathrm{fs}-4 \mathrm{ps}$ ) at $5 \mathrm{~Hz}$. The beam diameters of the FEL and the optical laser in the interaction volume were measured to be about $30 \mu \mathrm{m}$ and $50 \mu \mathrm{m}$, respectively.

As a first application of the experimental setup, the two-colour above threshold ionization (ATI) of rare gases has been investigated. The strong field of the optical laser can dramatically modify the photoelectron spectra when it is present in the interaction volume during the ionization process induced by the EUV FEL radiation. A typical spectrum recorded for complete overlap of the pulses is shown in figure 13 showing the $1 \mathrm{~s}$ photoelectron line of atomic He after photoionization with FEL radiation of $25.5 \mathrm{~nm}$ [71]. On both sides of the main photoelectron line so-called sidebands show up, which are separated by the photon energy of the optical laser and 
represent the direct two-photon process, i.e. the simultaneous interaction of the atom with both EUV and IR laser fields. The process, i.e. the intensity of the sidebands, is very sensitive to the overlap of both EUV and optical pulses, i.e. to the strength of the optical field, and so it can be used to monitor the degree of overlap of the IR and FEL pulses.

The soft-photon approximation or SPA [72] describes the process well and can be used to accurately predict both the strength of the sidebands as well as their sensitivity to the relative polarisation directions of the EUV and optical fields. The results of a SPA calculation are also plotted in figure 13 and are in very good agreement with the experiment for an optical dressing field of $2 \times 10^{11} \mathrm{~W} / \mathrm{cm}^{2}$.

When the laser field is increased further sidebands are produced representing the absorption and emission of many optical photons. The well-defined characteristics of both sources, and in particular their high degree of linear polarization is further used to get even greater insight into the dynamics of the photoionization process. In figure 14 the polarization dependence of the two-colour process is shown [73]. The spectra were recorded in the high field regime of the optical laser, i.e. with an average dressing field of about $6 \times 10^{11} \mathrm{~W} \mathrm{~cm}^{-2}$. Under these conditions up to three sidebands are clearly seen. Changing the relative orientation of the linear polarization vector of the optical laser with respect to that of the FEL, the intensity of the sidebands changes in a well defined, characteristic way. The highest intensity is observed when both polarization vectors are parallel and is conversely lowest when they are orthogonal to each other. For the case of low dressing fields, i.e. for IR laser intensities below $10^{11} \mathrm{~W} / \mathrm{cm}^{2}$, when only one sideband is seen on each side, this variation can theoretically be described within second order perturbation theory by the rather elegantly simple expression [73]: 


$$
\frac{d W}{d E}=3 S_{2}+\left(5 S_{0}+S_{2}\right) \cos ^{2} \theta
$$

where the coefficients $\mathrm{S}_{0}$ and $\mathrm{S}_{2}$ are related to the probability for emission of $s$ - and $d$ electrons, respectively, and $\theta$ is the relative angle between the linear polarization vectors.

The photoionization of the $1 \mathrm{~s}$ electron by two photons (EUV + NIR) leads to the ejection of a continuum electron with angular momentum $l=0$ (s-symmetry) or $l=2$ (d-symmetry). The given equation demonstrates that one can deduce from the measurement the ratio between $\mathrm{S}_{0}$ and $\mathrm{S}_{2}$, which is found to about 1.3, i.e., a higher probability for electron emission into the $\varepsilon s$ than into $\varepsilon d$ continuum. This result represents a clear deviation from the normal propensity rules in photoionization and is in contrast to the earlier results obtained by using high harmonic generation sources for investigations of the two-photon two-colour ionization of He via intermediate $\mathrm{He}^{*}$ 1 snp resonant states [74]. The analysis of the high field spectrum is significantly more challenging. The soft-photon model still describes well the observed tendencies, but the interpretation, i.e., the separation of the individual ionization channels is less straightforward since multiple ionization continua of higher and higher angular momenta have to be included in the calculations.

The manifestation of multiphoton processes is brought into sharper relief when a dressing field of more than $10^{14} \mathrm{~W} \mathrm{~cm}^{-2}$ is employed in these experiments. In this case a huge number of sidebands is produced and the main photoelectron line is almost completely suppressed (figure 15). The spectrum is now dominated by the influence of the optical field showing an increase in the electron kinetic energy of more than $20 \mathrm{eV}$, i.e the absorption of more than $12 \mathrm{Ti}$-Sapphire laser photons. The monochromaticity of the FEL radiation gives us unprecedented access to clean 
sidebands, free from interference with the signal from neighbouring harmonics $[75,76]$ or from a complicated Auger structure [77]. Hence, the intense FEL pulses mean that we can now begin to study optical multi-photon processes high up in the continuum of an atom (or indeed molecule) in an unperturbed fashion for the first time. The high optical field data shown in figure 15 and similar data for other rare gases are under analysis at the time of writing.

One can also exploit the strong optical field and resulting multi-photon processes combined with the high flux of the FEL to study EUV dissociation of molecules such as $\mathrm{H}_{2}$. After the ionization and subsequent dissociation of the molecule by the FEL pulse various atomic fragments are produced. Taking the specific case of excited neutral $\mathrm{H}$ atom product, the high optical field is used to ionize these excited states by multi-photon absorption. In a pump-probe arrangement the optical field is applied here to probe the population of the excited states and, potentially the dissociation dynamics of the molecular fragmentation. In figure 16 we show the photoelectron spectrum recorded with the output pulses of FLASH (operating at $13.5 \mathrm{~nm}$ ) and the Ti-Sa laser (operating at its fundamental wavelength of $800 \mathrm{~nm}$ ) overlapped. The strong signal due to ionization of excited atomic hydrogen $\left(\mathrm{H}^{*}, \mathrm{n}=2\right)$ is a clear indication of a multi-photon process since this ionization signal is observable only with the absorption of three $1.55 \mathrm{eV}$ photons. The strong signal suggests that there is possible resonant enhancement of the process. The two-photon excitation brings the electron into the region close to the ionization threshold and thereby also in the region of the Rydberg states, i.e., the resonant process is likely to occur. The additional lines related to the excited $n=3$ and $n=4$ levels correspond to a single photon absorption process. 
In a future extension of these experiments we intend to use the high intensity of the IR and EUV fields to purposely drive resonant processes, i.e., the two-colour field excitation energy (including any ponderomotive shift) is tuned to coincide with a resonant excitation of autoionising states (AIS). These processes are presently under study and offer many possibilities to obtain insight into the dynamics of inner-shell and correlated multi-electron excited AIS. Both the profile and position of such resonances depend rather critically on the intensity, wavelength and temporal detuning [78]. The resonances, which are reachable by two-colour excitation, can also be excited by a two-photon one-colour process. For example, the $3 \mathrm{~d}-4 \mathrm{~d}$ resonance of $\mathrm{Kr}$ at $92 \mathrm{eV}$ can be excited by combining two $46 \mathrm{eV}$ photons of the FEL [79]. The subsequent Auger decay and the related multi-photon processes can then be studied. Finally, the strong FEL pulses can also induce the direct two-photon process. This is in particular favorable for situation where already a huge cross section exists for the one-photon process, such as for the $4 \mathrm{~d}-\varepsilon \mathrm{f}$ excitation in $\mathrm{Xe}$ at about $90 \mathrm{eV}$. These twophoton one colour processes are presently under study and are expected to produce novel insights into the non-linear processes involving core electrons.

II.2.4 Toward imaging time-resolved molecular rearrangement using the photoelectron angular distribution of laser-aligned molecules (Amsterdam-AMOLF)

Observing chemical or physical processes on the atomic scale is a crucial step towards the understanding of molecular structure and dynamics. Consequently, the vision to perform coherent $\mathrm{x}$-ray diffractive imaging experiments to follow ultrafast structural dynamics on femtosecond timescales with sub-nanometer spatial resolution is one of the driving motivations behind the development of new X-ray Free Electron Lasers like LCLS or the European X-FEL. Proof-of-principle experiments of the application 
of this technique to micrometer [80] and nanometer sized [81] objects have already been performed with femtosecond soft-X-ray pulses from FLASH.

As an alternative approach to X-ray diffraction, one can perform diffractive imaging of molecules through electron scattering, using either externally generated electron pulses $[82,83]$ or using electrons ejected from within a molecule $[84,85]$. In the latter case, one exploits the fact that electrons leaving the molecule due to photoionization may scatter off one of the other nuclei, analogous to EXAFS measurements. The scattering processes encode diffractive information in the photoelectron angular distribution and can provide detailed insights when the photoelectron angular distribution is measured in the molecular frame. Moreover, if the electron emission can occur from multiple equivalent atoms within the molecule, additional interference patterns arise if the experiment is performed without a means to determine from which atom within the molecule the electron originates [86,87]. In both types of photoelectron emission considered here, i.e. electron diffraction off scattering centres and multi-centre interference between electron waves originating from different locations within the molecule, measured photoelectron angular distributions can give information about the positions of the individual nuclei and can be used as a probe of molecular structure.

In molecular imaging experiments of the type described above, it is a requirement that the measurements can be done in the molecular frame, i.e., in a coordinate system that is fixed with respect to the molecular axis. If not, most of the intensity variations that contain structural information will be averaged out. Information about the direction of the molecular axis can be obtained using a coincidence experiment [87] where electrons and ionic fragments are measured simultaneously, with the latter afterwards revealing the alignment in the laboratory 
frame at the time of ionization (COLTRIMS or reaction microscope REMI [33]). Challenges to such an experiment are the fact that it requires that the molecule fragments and that the axial recoil approximation is valid, while furthermore the approach is experimentally challenging since only few coincident events can be measured per laser shot, making it difficult to use these techniques at a moderate repetition rate machine such as an FEL. Coincident electron-ion detection can be avoided making use of laser-induced alignment to manipulate the angular distribution of molecular samples in combination with the use of a velocity map imaging spectrometer [88] (VMIS), which permits the measurement of kinetic and angular distributions of electrons or ions with high count rates. Laser-induced alignment techniques exploit the interaction of the laser field with the molecular polarizability and allow the measurements to be done directly in the molecular frame [89]. Both adiabatic alignment [90], where molecules are exposed to a slowly varying laser field, and non-adiabatic (or impulsive/field-free) alignment [91,92], where molecules undergo periodic alignment after receiving a kick by a short laser pulse, have been successfully implemented.

Here, we report on experiments performed at FLASH aimed at using aligned molecules and VMI detection to follow molecular dynamics, such as dissociation, by studying interference and diffraction of photoelectrons emitted through single-photon ionization by the EUV/soft X-ray FEL pulse. The first realization of field-free alignment at FLASH, making use of $\mathrm{CO}_{2}$ molecules, will be discussed as well as a first attempt to observe time-dependencies in the molecular frame photoelectron distribution of dissociating $\mathrm{Br}_{2}$ molecules. In order to perform these experiments a pump-pump-probe arrangement has been developed, where a first optical pump laser pulse is used to initiate the alignment dynamics, a second pump laser pulse (not used 
in the case of $\mathrm{CO}_{2}$ ) initiates dynamics in the molecules under investigation, and the role of the probe laser is played by the FLASH FEL, at a variable time delay with respect to the other two lasers.

One of the most challenging aspects of doing a pump-probe experiment at FLASH is the temporal and spatial overlap between an optical laser pulse and the EUV-FEL pulse. This was achieved in the past using sideband generation in photoelectron spectra $[69,71]$. However, this can be quite tedious since the spatial overlap can only be established close to temporal overlap, which can furthermore be compromised by the $600 \mathrm{fs}$ time jitter [71] between the FLASH pulse and available synchronized Ti:Sapphire laser pulses. In our experiment, temporal and spatial overlap was established using a newly developed technique making used of the VMI detector in the so called "spatial imaging mode" combined with the observation of bond-softening in $\mathrm{H}_{2}$ molecules (i.e. dissociation of $\mathrm{H}_{2}{ }^{+}$molecules ions that are formed by the FLASH FEL, making use of a moderately intense IR beam). Bondsoftening only requires that the EUV pulse arrives before the IR pulse in order to observe the signal at spatial overlap. Once spatial overlap is achieved, it is therefore straightforward to scan the delay to find the temporal overlap (see figure 17(a-d)). The spatial imaging mode of the VMI detector allows the observation of the beam propagation in the $2 \mathrm{D}$ plane defined by the detector. By setting the position of the IR beam so that it overlaps with the FEL beam in the observed images (see for instance figure $17(\mathrm{e})$ ), only the relative position of the two beams along the direction perpendicular to the detector plane is unknown. However, this can easily be fixed by looking at the difference in the time-of-flight of fragments ejected by the IR pulse and by the FEL pulse. By moving the IR beam so that the arrival time of the IR-induced and the EUV-induced fragments coincide, spatial overlap is readily achieved. This 
method allows therefore for an easy and fast overlap of the two beams, both in time and space.

As a first step toward imaging time resolved molecular dynamics using the diffractive imaging concept introduced before, we performed proof-of-principle molecular alignment experiments, where a field-free sample of aligned molecules was prepared using the FLASH in-house femtosecond Ti:Sapphire IR laser. The degree of alignment was subsequently measured by using the VMI to record the $2 \mathrm{D}$ momentum distribution of $\mathrm{O}^{+}$fragments resulting from Coulomb explosion of $\mathrm{CO}_{2}{ }^{2+}$ ions created by photoionization with $46 \mathrm{eV}$ FLASH photons. Figure $18(\mathrm{a}-\mathrm{d})$ shows the recorded $\mathrm{O}^{+}$fragments distribution for different time delays between the optical laser pulse and the FEL pulse as well as the time evolution of $<\cos ^{2} \theta>$ obtained from angular integration of the images. $\theta$ is defined as the angle between the laser polarization and the direction of the ejected fragment. In the axial recoil approximation, i.e. if the dissociation is faster than the rotational dynamics, the direction of the ejected fragments is equivalent to the orientation of the molecular axis and can therefore be used to quantify the degree of molecular alignment. As shown in this figure, when the FEL pulse precedes the IR pulse, the angular distribution of the $\mathrm{O}^{+}$fragment is almost isotropic. This is no longer the case at time overlap where the angular distribution peaks along the polarization of the IR pulse, which indicates a strong alignment of the molecules. A periodic narrowing of the angular distribution is also observed at later time delays, which is characteristic of field-free molecular alignment. The interaction with the short laser pulse leads to the formation of a rotational wave packet which rephases periodically in time, leading to an alignment of the molecule after the pulse has ended. For $\mathrm{CO}_{2}$, revivals of the alignment are expected every $\mathrm{T}_{\mathrm{r}} / 4=10.2 \mathrm{ps}$, where 
$\mathrm{T}_{\mathrm{r}}$ is defined as the rotational period of the molecule. This time dependence is clearly reproduced in the extracted time evolution of $\left\langle\cos ^{2} \theta>\right.$ (figure 18(e)).

Following this successful demonstration of dynamic alignment and the probing of static molecules, a three-pulse experiment was set up at FLASH. An 800 $\mathrm{nm}$ laser pulse from the in-house femtosecond Ti:Sapphire laser was used to align Bromine molecules. This pulse was followed by a second, $400 \mathrm{~nm}$, pump pulse to induce dissociation of the neutral molecule. Finally, the dissociation dynamics was probed through EUV ionization by $13 \mathrm{~nm}$ FEL pulses. Figure 19(a-c) shows 2D momentum maps of the resulting $\mathrm{Br}^{2+}$ fragments in the presence of FEL only (a), with both the $400 \mathrm{~nm}$ and the FEL beam (b) and when all three pulses are present (c). In the presence of the FEL pulse, the 2D velocity distribution is composed of concentric rings originating from dissociative ionization and Coulomb explosion of the molecule. The new contribution observed in figure 19(b) results from the ionization by the FEL pulse of fragments of the dissociation initiated by the $400 \mathrm{~nm}$ pulse. When adding the $800 \mathrm{~nm}$ pulse, the angular distribution peaks along the laser polarization similar to the $\mathrm{CO}_{2}$ experiment, which indicates that the molecules are aligned prior to dissociation and ionization. A very interesting feature is observed in the time evolution of the kinetic energy release of the $\mathrm{Br}^{2+}$ fragments, when the delay between the $800 \mathrm{~nm}$ and the $400 \mathrm{~nm}$ lasers is fixed and the delay of the FEL pulse is changed (figure 19(d)). A sharp contribution evolving from $7 \mathrm{eV}$ to $1.8 \mathrm{eV}$ on a 2 ps timescale is observed that arises from molecules that are ionized at different points along the dissociation curve and which therefore end-up at different positions along the accessible Coulomb explosion channels by the EUV pulse. This leads to a decrease of the $\mathrm{Br}^{2+}$ kinetic energy release as the internuclear distance increases along the dissociation curve. The results shown in figure 19(c) satisfy almost all the requirements that one can define 
for an experiment aimed at using molecular frame photoelectron angular distributions as a means to study time-dependent molecular dynamics, namely the availability and implementation of (i) velocity map imaging, (ii) dynamic alignment, (iii) the implementation of a pump-pump-probe arrangement and (iv) the availability of fragment ion data where the strongest contribution corresponds to the channel of interest for the photoelectron measurement. Hence, first attempts were made to record photoelectron angular distributions under these conditions, with the aim of identifying effects due to photoelectron interference and/or diffraction within the dissociating molecule. First photoelectron measurements showed a large contribution from background due to scattered EUV photons impinging on the detector, however after designing and adding entrance and exit apertures to the spectrometer, high-quality photoelectron momentum distributions with high count rates (see figure 20(b)) could be recorded. Unfortunately, due to the large time jitter between the FEL pulse and the in-house femtosecond Ti:Sapphire laser pulses combined with the expected short dissociation dynamics ( $<80 \mathrm{fs}$ ), the dissociation of $\mathrm{Br}_{2}$ molecules could not be resolved yet. However, in future planned experiments, using a newly developed timing electro-optical sampling system that permits the measurement of on a singleshot basis the timing between the optical laser and the FEL pulse with sub-50 fs resolution, all conditions will exist to resolve fast molecular rearrangement through EUV photoelectron interference and/or diffraction.

\section{II.3 First experiments at LCLS}

\section{II.3.1 Opportunities for AMO physics at LCLS (Stanford-SLAC)}

The Linac Coherent Light Source (LCLS), the world's first hard x-ray laser first lased on April 10, 2009. The FEL was built at the SLAC National Accelerator Laboratory 
in Menlo Park, CA utilizing the last third of the existing $3 \mathrm{~km}$ long linac originally constructed in 1962. A photocathode electron gun and short S-band injector linac were installed at the $2 / 3$ point along the linac to inject electrons at $135 \mathrm{MeV}$ into the existing SLAC linac [93]. Two bunch compressors, the first at a point where the electron bunch has been accelerated to $250 \mathrm{MeV}$ and the second where the electron beam energy is $4.3 \mathrm{GeV}$, were also installed to produce the very high current density electron bunch required to support lasing at $1.5 \mathrm{~nm}$. The linac downstream of the 2 nd bunch compressor can be used to further accelerate the electron bunch up to $13.6 \mathrm{GeV}$ if required before it is transported to the undulators. Repetition rates of up to $120 \mathrm{~Hz}$ can be provided by the electron gun and linear accelerator, although during the first experimental run the LCLS was operated at $30 \mathrm{~Hz}$. A set of up to $333.4 \mathrm{~m}$ long undulators with a magnetic period of $3 \mathrm{~cm}$ can be positioned on the electron beam to generate the FEL radiation from the extremely bright electron beam from the linac [94].

The LCLS performed marvellously during the first experimental run OctoberDecember 2009 providing users with a variety of wavelengths, pulse durations and intensities. In order to change the wavelength of the FEL radiation, the electron beam energy must be changed as the undulators are built with a fixed gap, a process that was accomplished in a few minutes or a few tens of minutes depending on the magnitude of the change. Photon energies between $770-2000 \mathrm{eV}$ were used during the first experimental run, although the range will be extended to approximately 570$10000 \mathrm{eV}$ in the next period. Pulse durations of $\sim 80-300 \mathrm{fs}$ were provided in the normal operating mode of the LCLS where an electron bunch charge of $250 \mathrm{pC}$ is accelerated and delivered to the undulators. In this configuration, pulse energies of $1-3.5 \mathrm{~mJ}$ were typically produced, depending on the pulse duration and the tune of the machine. A 
low charge mode, producing pulses $<10 \mathrm{fs}$ in duration was introduced during the user run [57]. In this mode 20 or $40 \mathrm{pC}$ electron bunches were accelerated into the undulators producing FEL pulses of $0.1-0.3 \mathrm{~mJ}$. Variations in the pulse duration in the 80-300fs regime were accomplished in minutes by varying the compression of the second bunch compressor, while changing from normal to low current mode, and hence from long to short pulse, required as much as four hours of effort by the accelerator operators. Stability of the beam, both in position and intensity, was typically on the order of $10-15 \%$ rms with periods of both better and poorer performance during this first run. Overall, the LCLS beam proved to be both more versatile in the available parameters and more stable in performance than initially expected, supporting a very successful first experimental run in late 2009 .

The experimental area at the LCLS consists of six experimental hutches divided between two experimental halls, the near experimental hall beginning approximately $100 \mathrm{~m}$ downstream from the end of the undulators and the far experimental hall, an additional 250m further downstream. Each hutch has a designated scientific thrust area and is being equipped with instrumentation to carry out the first experiments. The first two hutches are dedicated to soft x-ray experiments owing to the $2 \mathrm{keV}$ cut-off of the mirrors used to deflect the beam away from the straight ahead direction and are intended for atomic, molecular and optical (AMO) science and soft x-ray materials research (SXR) respectively. The third hutch, which is also in the near experimental hall, is intended for x-ray pump and probe (XPP). In the far experimental hall the experiments, in order, are x-ray correlation spectroscopy (XCS), coherent x-ray imaging (CXI) and materials under extreme conditions (MEC). During the first experimental run in 2009 only the AMO hutch was operational and it's instrument is described further below. 
Instrumentation for AMO science at the LCLS was designed to capitalize on the unprecedented high intensity and short duration of the pulses of FEL x-ray radiation produced by this new source to study multiphoton x-ray and time-resolved processes $[5,95]$. In order to preserve the peak intensity of the LCLS, the AMO instrument uses the FEL beam without further monochromatisation, utilizing the natural narrow bandwidth of the FEL beam of about $0.5 \%$ [95]. After a set of three planar deflection mirrors, each with an angle of incidence of $13.85 \mathrm{mrad}$ and located in the front-end enclosure (FEE) immediately upstream of the hutch, the FEL beam enters the hutch. Focusing mirrors are used to produce a small waist and hence extremely high x-ray intensity in the interaction region. In order to accommodate various end-stations at different distances from the optics, the mirrors are dynamically bent $400 \mathrm{~mm}$ long $\mathrm{B}_{4} \mathrm{C}$ coated polished silicon flats [96]. When focused to the interaction region in the main chamber, a beam waist of $1.5 \mu \mathrm{m}$ was measured by imprinting the beam in a solid paddle. At this size, $\mathrm{x}-$ ray intensities of $10^{18} \mathrm{~W} / \mathrm{cm}^{2}$ can be produced and used to investigate strong-field and multiphoton effects. Instrumentation downstream of the focusing optics is separated into two chambers, the "high field physics" (HFP) chamber and "diagnostics" chamber, each of which can operate in concert or independently. The HFP chamber delivers gaseous samples to the interaction region in a pulsed skimmed supersonic gas jet. The gas beam crosses the FEL beam in the extraction region of an ion time-of-flight spectrometer. Arrayed around this interaction region are a set of five high-resolution electron time-of-flight spectrometers that have been used to measure photo- and Auger electrons. The diagnostics chamber houses a magnetic bottle electron spectrometer designed to efficiently collect electrons and provide single-shot electron spectra. Gaseous samples are introduced into this chamber through an effusive gas needle. A YAG 
crystal at the downstream end of the diagnostics chamber was used to align the chambers and monitor the position stability of the FEL beam. A pulse energy monitor was also included in the diagnostics chamber, although it was not commissioned in the first experimental run. A synchronized Ti:Sapphire laser can deliver $2 \mathrm{~mJ}$ pulses of $800 \mathrm{~nm}$ light as short as $30 \mathrm{fs}$ to either chamber for experiments.

The AMO instrumentation saw first light on Aug. 182009 and was commissioned over the following six weeks. During this period, the operation of each of the instruments was verified and characterized. Immediately following the commissioning period, ten weeks of user experiments were conducted and some of the results from one of them are reported below. During the last four weeks of the experimental run, the diagnostics chamber was removed and replaced with a user supplied chamber with unique capabilities. While this mode of operation is not encouraged, due primarily to the complexity of data acquisition at beam rate and possible damage to equipment from the focused FEL beam, certain unique applications can be supported with sufficient planning. Experimental runs will be scheduled every six months and with each new experimental run, an additional LCLS experiment will be commissioned and added to the suite of available instruments. This will expand the scientific capabilities of the LCLS but also reduce the amount of time dedicated to each scientific theme. To alleviate the overwhelming demand anticipated, plans are already underway to expand the capacity of the LCLS with the addition of another undulator.

\section{II.3.2 Photoionization of $N_{2}$ using Short and Intense LCLS X-Ray Pulses (Western Michigan University)}

The study of atomic and molecular inner-shell photoionization with conventional xray sources allowed the investigation of single-photon absorption and the 
understanding of single core holes ionization $[97,98]$. These studies enabled a rich physics dominated by electron correlation phenomena.

Short x-ray pulses from free electron lasers open a new field dominated by multi-photon ionization and favouring, if the intensity is high enough, multiple core hole studies in atoms, molecules [99], solids and biomolecules. As the first x-ray FEL, the intense x-ray short pulses from the Linac Coherent Light Source (LCLS), the first X-ray FEL, provides a powerful tool for investigating the creation and decay of multiple core-holes.

First experiments using the LCLS investigated the response of simple diatomic molecules, such as $\mathrm{N}_{2}$, to intense, short pulses. Multiple ionization was studied by examining the electronic relaxation and fragmentation of the excited molecule. In particular, the Auger and secondary electron relaxation pathways subsequent to double core vacancies, as well as the fragmentation patterns and charge-state distributions of the resulting ions were measured. Although the analysis is still underway, we expect that the characterization of the complex molecular ionization and dissociation dynamics enabled by the LCLS will provide new insight into the correlated motion of the electrons remaining in the targets and into fundamental aspects of ultrafast molecular physics and chemistry.

The LCLS provided short laser pulses from 7 fs to 280 fs with photon energies up to $2 \mathrm{keV}$ at the AMO beamline. The $\mathrm{x}$-ray beam was focused by a set of Kirkpatrick-Baez (KB) mirrors to about $1 \mu \mathrm{m}$ diameter focal point to obtain an intensity of up to $10^{18} \mathrm{~W} \mathrm{~cm}^{-2}$. These intensities are sufficient to investigate multiphoton and multiple ionization processes. The AMO beamline and instrument allowed the detection of both ion fragments and electrons. 
The ion spectra were taken with an ion time-of-flight (TOF) spectrometer, the resolving power of which was sufficient to separate all highly ionized fragments. We have observed the multiple ionization of $\mathrm{N}_{2}$ resulting in fragment ions of up to bare $\mathrm{N}^{7+}$. Figure 21 shows the ion TOF spectrum, demonstrating different charge states. For all charge states, the ion yields increase as the intensity of the x-ray beam increases. At the intensities achievable in the experiments, except the lowest one, fully stripped $\mathrm{N}$ atomic ions $\left(\mathrm{N}^{7+}\right)$ are detected. The data, which is presently being analyzed, will lead to an understanding of the response of $\mathrm{N}_{2}$ to different short pulse duration and pulse intensity [100].

\section{Conclusions and Future Perspectives}

The field of non-linear optics has been flourishing for more than four decades, gaining the right in that time interval to claim at least one direct Nobel Prize in 1981 in the form of Arthur Schalow and Nicolaas Bloembergen [101]. This field is so well established that it is becoming applications directed and hugely important in biomedical 'theranostics' with real societal benefits such as oncological multiphoton imaging for cancer cell detection [102] and therapeutic intervention [103], not to mention its application in sub-cellular imaging [104]. This 'conventional non-linear optical' domain concerns only the response of valence or outer-shell electrons driven by intense and coherent optical laser fields - in effect non linear optics has to date been limited to accessing only weakly bound electrons.

In this respect, LCLS and the coming generation of X-ray FELs such as the European XFEL [105] and the next phase of SCSS in Japan [4] are enormously exciting. Already LCLS can produce multi-millijoule pulses in programmable durations from a few to a few hundred femtoseconds. As pulse energies are pushed 
higher and pulse widths enter the hundred attosecond timescale, the first petawatt Xray lasers will be approached. Already, at LCLS, fields of $10^{18} \mathrm{~W} \mathrm{~cm}^{-2}$ are readily available [5] and the analysis of evidence for multiphoton multi-core electron ejection is underway - effectively multiphoton 'hollowing out' of atoms right down to the $\mathrm{K}$ shell. 'Photogenerated' hollow atoms [e.g., 106, 107] and ions [e.g., 108] have only been observed to date in single photon excitation where the multiply excited electrons are coupled via an inherent strong electron correlation. A first experiment with a cold Li target to study strongly correlated phenomena in the double ionization of state prepared lithium has already been performed at FLASH [109] and future experiments on a host of (initial) state prepared multiphoton-generated hollow atoms and ions are within reach.

As this topical review emphasises, the new generation of SASE FELs opens up the opportunity to explore new pathways to the non-linear behaviour of matter involving not just valence electrons as heretofore, but also, for the very first time, inner shell electrons. Hence truly ultrafast processes involving internal rearrangement/relaxation, multielectron shake off, multiphoton-multielectron excitation/decay, etc. all exclusive to this domain are becoming available to scientific scrutiny.

What appears really exciting is the crossover from conventional linear or weak field EUV and X-ray AMO physics, which has brought deep insights to materials and biomedical sciences, to non-linear short wavelelength processes. X-ray FELs operate at wavelengths comparable to the atomic unit of spatial scale and with pulse durations that are already approaching the atomic unit of time. Such XFELs will permit atomic structure and dynamics to be directly and simultaneously probed on their intrinsic space and timescales. Already impressive results on static [80] and dynamic [110] 
nanoscale imaging have been demonstrated at FLASH. One can speculate that ultimately the atomic nature of systems ranging from cancer causing rogue molecules to complex nanostructures may be distinguished, extracted and possibly even controlled. For example, the intensity that SASE FELs bring to the problem means one can begin to think in terms multiphoton X-ray imaging of irradiated cancer cells during X-ray therapeutic intervention, perhaps combined with photo/Auger electron biochemical diagnostics - in effect 'X-ray theranostics'.

But first one must obtain the fullest understanding of the interaction of intense EUV and X-ray laser radiation with the simplest components of matter, i.e., atoms and molecules. To date we have touched on the possibilities that short wavelength free electrons lasers offer to explore fundamental light-matter interactions in their simplest forms. We have translated, with some success, techniques from conventional synchrotron and intense AMO physics that have provided us with important insights into the behaviour of prototypical atoms and molecules in electromagnetic fields of unprecedented intensity with ultrashort cycle times (attoseconds). Before we can realise the true potential of these sources we need to continue to explore their nonlinear interactions with target systems that we understand and to adopt existing laser techniques such as seeding $[111,112]$ to achieve full phase coherence as in e.g., the sFLASH project [113]. Indeed, should current successes in a related field, namely Xray harmonics from relativistic plasma based 'oscillating mirrors' [114, 115] continue to make progress, seeded X-ray FELs could become a reality. In addition we can approach multiphoton coherent process control in the EUV and X-ray regime and only then can one confidently move to 'real world' applications and realise the true potential of FELs to impact our lives as positively as conventional lasers and nonlinear processes have over the past five decades. 


\section{Acknowledgements}

K. U. and H. F. acknowledge the support for the X-ray Free Electron Laser Utilization Research Project of the Ministry of Education, Culture, Sports, Science and Technology of Japan (MEXT). MR, AS and KT wish to thank Sergey V. Bobashev (Ioffe Institute, St. Petersburg), Bernd Sonntag (University of Hamburg) and Miron Ya. Amusia (Ioffe Institute, St. Petersburg and Hebrew University, Jerusalem) for helpful discussions and Torsten Feigl fo IOF (Jena) for the multilayer mirror. Work funded under DFG project no. RI 804/5-1. The work of J. U., R. M., A. R. and Y. H. J. was supported by the Max-Planck Initiative DESY FEL (MIDFEL) and the MaxPlanck Advanced Study Group (ASG) at the Center for Free Electron Laser Science (CFEL) at Hamburg. They are grateful to A. Belkacem and LBNL staff, J. Verhoeven from AMOLF as well as E. Louis and F. Bijkerk (FOM) for designing and providing the multi-layer mirror. The work would not have been possible without the sustained and enthusiastic support by the DESY accelerator team and beam line scientists, especially R. Treusch. Y.H.J. acknowledges support from DFG project nr. JI 110/2-1, E.P, J.F.P.T. We thank our collaborators from the University of Frankfurt, MPQ Garching, Tohoku University Sendai, GANIL, University of Crete, University of Missouri Rolla and KSU. Finally, we appreciated theoretical help from F. Martin, A. Voitkiv, A. Kheifets, B. McCurdy, J. Feist, J. Burgdörfer, S. Fritzsche, N. Kabachnik as well as discussions with P. Lambropoulos, R. Shakeshaft, B. Piraux, K. Bartschat and many others. The work of J. C., M. M. and E. T. K. is supported variously by the Ulysses France-Ireland programme, EU COST Action MP0601, Science Foundation Ireland (Grant Nos. PHY041 and 07/IN.1/I1771) and the HEA PTRLI 4-INSPIRE programme. They would also like to acknowledge the contributions of many graduate students and postdoctoral fellows and especially collaborators, D. Cubaynes (LIXAM, Orsay), A. Maquet, E. Taieb (LCPMR, Paris) and A. Grum-Grzhimailo (Moscow State University). P. J. acknowledges support from the Swedish Research Council. The work of A.R, P.J. and M.V was part of the research program of the "Stichting voor Fundamenteel Onderzoek der Materie (FOM)", which is financially supported by the "Nederlandse organisatie voor Wetenschappelijk Onderzoek (NWO)". A. R., P. J. and M. V. also acknowledge travel support from the European Community under Contract RII3-CT-2004-506008 (IA-SFS). N. B., L. F. and M. H. would like to acknowledge support from DOE-SC-BES, Chemical Sciences, Geosciences, Biosciences Division. MH thanks the Humboldt Foundation for his Feodor Lynen Fellowship.

\section{References}

[1] J. Andruszkow, B. Aune, V. Ayvazyan et al. Phys. Rev. Lett 853825 (2000).

[2] V. Ayvazyan, N. Baboi, J. Bahr et al., Eur. Phys. J. D 37297 (2006).

[3] W. Ackermann, G. Asova, V. Ayvazyan et al., Nature Photon. 1336 (2007).

[4] T. Shintake, H. Tanaka, T. Hara et al., Nature Photon. 2555 (2008).

[5] J. D. Bozek, Eur. Phys. J. ST. 169129 (2009).

[6] C. Pellegrini and S. Reiche, IEEE J. Select. Topics in Quant. Electron. 10 (2004).

[7] J. Feldhaus, J. Arthur and J. B. Hastings, J. Phys. B:At. Mol. Opt. Phys. 38 S799 (2005).

[8] T. Shintake, AIP Conf. Proc. 879228 (2007).

[9] Z. Huang and K-J. Kim, Phys. Rev. ST. Accel. Beam. 10034801 (2007).

[10] S. Khan, J. Mod. Opt. 553469 (2008). 
[11] J. T. Costello, J. Phys. Conf. Ser. 88012057 (2007).

[12] C. Bostedt, H. N. Chapman, J. T. Costello et al., Nucl. Instrum. Meth. A 601108 (2009).

[13] M. Protopapas, C. H. Keitel and P. L. Knight, Rep. Prog. Phys. 60389 (1997).

[14] P. Agostini and L. F. DiMauro, Rep. Prog. Phys. 67813 (2004).

[15] L. V. Keldysh, Sov. Phys. JETP 201307 (1965).

[16] J. Rossbach, Nucl. Instrum. Meth. A 375269 (1996).

[17] P. Lambropoulos and P. Zoller, Phys. Rev. A 71379 (1981).

[18] P. Lambropoulos, L. A. A. Nikolopoulos, M. G. Makris et al., Phys. Rev. A 78 055402 (2008).

[19] S. Fritzsche, A. N. Grum-Grzhimailo, E. V. Gryzlova et al., J.Phys. B: At. Mol. Opt. Phys. 41165601 (2008).

[20] T. Sato, T. Okino, K. Yamanouchi et al., Appl. Phys. Lett. 92154103 (2008).

[21] K. Ueda and J. H. D. Eland, J. Phys. B 38 S839 (2005).

[22] R. Dörner, Th. Weber, M. Weckenbrock et al., Multiple Ionization in Strong Laser Fields in: Advances in Atomic and Molecular Physics, ed: B. Bederson and H. Walther (Academic Press 2002) Volume 48 pp 1-36.

[23] K. Motomura, L. Foucar, A. Czasch et al., Nucl. Instrum. Meth. A 606770 (2009).

[24] K. Motomura, H. Fukuzawa, L. Foucar et al. J. Phys. B: At. Mol. Opt. Phys. 42 221003 (2009).

[25] H Fukuzawa, K Motomura, X-J Liu et al., J. Phys. B: At. Mol. Opt. Phys. 42 181001 (2009).

[26] A. Yamada, H. Fukuzawa, K. Motomura et al., submitted.

[27] H. Fukuzawa X.-J. Liu, G. Prümper et al., Phys. Rev. A 79031201 (2009).

[28] H. Iwayama, K. Nagaya, M. Yao et al., J. Phys. B: At. Mol. Opt. Phys. 42 134019 (2009).

[29] H. Fukuzawa X.-J. Liu, G. Prümper et al., J. Phys. Conf. Ser. (ICPEAC 2009 proceedings) in press.

[30] H. Iwayama, K. Nagaya, M. Yao et al, J. Phys. Conf. Ser. (Correlation and Polarization 2009 proceedings) in press.

[31] X.-J. Liu, H. Fukuzawa, G. Prümper et al., Rev. Sci. Instrum. 053105 (2009).

[32] G. Prümper, H. Fukuzawa, T. Lischke et al, Rev. Sci. Instrum. 78083104 (2007).

[33] J. Ullrich, R. Moshammer, A. Dorn et al., Rep. Prog. Phys. 661463 (2003).

[34] O. Jagutzki, A. Cerezo, A. Czasch et al., IEEE Transact. Nucl. Sci. 492477 (2002).

[35] A. Czasch, L.Ph.H. Schmidt, T. Jahnke et al., Phys. Lett. A 34795 (2005).

[36] G. Da Costa, F. Vurpillot, A. Bostel et al., Rev. Sci. Instrum. 76013304 (2005).

[37] L. Foucar, Ph. D. thesis, J. W. Geothe University, Frankfurt/Main (2008).

[38] K. Tiedtke, A. Azima, N. von Bargen et al. New J. Phys. 11023029 (2009).

[39] A. A. Sorokin et al., Phys. Rev. Lett. 99213002 (2007).

[40] M. Richter, M. Y. Amusia, S. V. Bobashev et al., Phys. Rev. Lett. 102163002 (2009).

[41] M. G. Makris, P. Lambropoulos and A. Mihelic, Phys. Rev. Lett. 102033002 (2009).

[42] VUV and Soft X-Ray Photoionization, edited by U. Becker and D. A. Shirley (Plenum Press, New York, 1996), and references therein

[43] Giant Resonances in Atoms, Molecules, and Solids, edited by J. P. Connerade, J. M. Esteva and R. C. Karnatak (Plenum Press, New York, 1987), and references therein 
[44] N. Delone and V. Krainov, Multiphoton Processes in Atoms (Springer, New York, 2000).

[45] A. A. Sorokin, S. V. Bobashev, K. Tiedtke et al., J. Phys. B 39 L299-L304 (2006).

[46] R. Moshammer, Y. H. Jiang, L. Foucar et al., Phys. Rev. Lett. 98 203001(2007).

[47] A. A. Sorokin, M. Wellhofer, S. V. Bobashev et al., Phys. Rev. A 75 051402(R) (2007).

[48] M. Nagasano et al., Phys. Rev. A 75 051406(R) (2007).

[49] Y. Nabekawa, H. Hasegawa, E. J. Takahashi et al., Phys. Rev. Lett. 94043001 (2005).

[50] H. Hasegawa E. J. Takahashi, Y. Nabekawa et al., Phys. Rev. A 71023407 (2005).

[51] L. Feng and H.W. van der Hart, J. Phys. B 36 L1 (2003).

[52] S. Laulan and H. Bachau, Phys. Rev. A 68013409 (2003).

[53] E. Foumouo, G. L. Kamta, G. Edah et al., Phys. Rev. A 74063409 (2006).

[54] L. A. A. Nikolopoulos and P. Lambropoulos, J. Phys. B 401347 (2007).

[55] I. A. Ivanov and A.S. Kheifets, Phys. Rev. A 75033411 (2007).

[56] J. Feist, S. Nagele, R. Pazourek et al., Phys. Rev. A 77043420 (2008).

[57] Y. Ding, A Brachmann, F. J. Decker et al., Phys. Rev. Lett. 102254801 (2009).

[58] D. Horner, C. W. McCurdy and T. N. Rescigno, Phys. Rev. A 78043416 (2008).

[59] J. Feist, R. Pazourek, S. Nagele et al, J. Phys. B: At. Mol. Opt. Phys 42134014 (2009).

[60] X. Guan, K. Bartschat and B. I. Schneider, Phys. Rev. A 77043421 (2008).

[61] E. Foumou, P. Antoine, B. Piraux et al., J. Phys. B: At. Mol. Opt. Phys 41 051001 (2008).

[62] A. Rudenko, L. Foucar, M. Kurka et al., Phys Rev. Lett. 101073003 (2008).

[63] M. Kurka et al., (submitted for publication)

[64] M. Kurka, A. Rudenko, L. Foucar et al., J. Phys. B: At. Mol. Opt. Phys 42 141002 (2009).

[65] Y. H. Jiang, A. Rudenko, M. Kurka et al., Phys. Rev. Lett. 102123002 (2009).

[66] Y. H. Jiang, A. Rudenko, E. Plesiat et al., Phys. Rev. A 81021401 (2010).

[67] Y. H. Jiang et al., (submitted for publication)

[68] E. Gagnon, P. Ranitovic, X. M. Tong et al., Science 3171374 (2007) .

[69] M. Meyer, D. Cubaynes, P. O'Keeffe et al., Phys. Rev. A 74011401 (2006).

[70] P. Radcliffe, S. Duesterer, A. Azima et al., Nucl. Instrum. Meth. A 583516 (2007).

[71] P. Radcliffe, S. Duesterer, A. Azima et al., Appl. Phys. Lett. 90, 131108 (2007).

[72] A. Maquet and R. Taieb, J. Mod. Opt. 541847 (2007).

[73] M. Meyer, D. Cubaynes, D. Glijer et al., Phys. Rev. Lett. 101193002 (2008).

[74] A. Johansson, M. K. Raarup, Z. S. Li et al., Eur. Phys. J. D 223 (2003).

[75] Guyetand O, Gisselbrecht M, Huetz A, et al. J. Phys. B: At. Mol. Opt. Phys 41 051002 (2008).

[76] P. O’Keeffe, R. Lopez-Martens, J. Mauritsson et al., Phys. Rev. A 69 051401(R) (2004).

[77] J. M. Schins, P. Breger, P. Agostini et al., Phys. Rev. Lett. 732180 (1994).

[78] S. I. Themelis, P. Lambropoulos and M. Meyer, J. Phys. B: At. Mol. Opt. Phys 374281 (2004).

[79] M. Meyer, D. Cubaynes, V. Richardson et al., (submitted for publication)

[80] H. N. Chapman, A. Barty, M. J. Bogan et al. Nat. Phys. 2839 (2006).

[81] M. J. Bogan, W. H. Benner, S. Boutet et al., Nano Letters 8310 (2008). 
[82] H. Ihee, V. A. Lobastov, U. M. Gomez, et al., Science 291458 (2001).

[83] B. J. Siwick, J. R. Dwyer, R. E. Jordan et al., Science 3021382 (2003).

[84] M. Meckel, D. Comtois, D. Zeidler et al., Science 3201478 (2008).

[85] A. Landers, T. Weber, I. Ali et al., Phys. Rev. Lett. 87013002 (2001).

[86] B. Zimmermann, D. Rolles, B. Langer et al., Nat. Phys. 4649 (2008).

[87] D. Rolles, M. Braune, S. Cvejanovic et al., Nature 437711 (2005).

[33] J. Ullrich, R. Moshammer, A. Dorn et al., Rep. Prog. Phys. 661463 (2003).

[88] P. Johnsson, W. Siu, A. Gijsbertsen et al., J. Mod. Opt. 552693 (2008).

[89] H. Stapelfeldt and T. Seideman, Rev. Mod. Phys. 75543 (2003).

[90] H. Sakai, C. P. Safvan, J. J. Larsen et al., J Chem. Phys. 11010235 (1999).

[91] F. Rosca-Pruna and M. J. J. Vrakking, Phys.Rev. Lett. 8153902 (2001).

[92] P. Johnsson et al., J Phys. B 42134017 (2009).

[93] R. Akre, D. Dowell, P. Emma et al., Phys. Rev. ST Accel. Beam. 11030703 (2008).

[94] E. Gluskin, N. A. Vinokurov, G. Decker et al., Nuc. Instr. Meth. Phys. Res. A 475323 (2001).

[95] H.-D. Nuhn, Nuc. Instr. Meth. Phys. Res. A 429249 (1999).

[96] M. R. Howells, D. Cambie, R. M. Duart et al., Optical Engineering 392748 (2000).

[97] M. S. Schoffler, N. Petridis J. Titze et al., Science 320920 (2008).

[98] D. Rolles, H. Zhang, Z. D. Pesic et al., Chem. Phys. Lett. 468148 (2009).

[99] L. S. Cederbaum, F. Tarantelli, A. Sgamellotti et al., J. Chem. Phys. 856513 (1986).

[100] L. Fang, M. Hoener, C. Blaga et al. (to be published).

[101] N. Bloembergen, Rev. Mod. Phys. 54685 (1982).

[102] X. C. Qu, J. Wang, Z. X. Zhang et al., J. Biomed. Opt. 13031217 (2008).

[103] S. M. Z. Hossain, S. M. G. Azam \& S. M. E Babar, Mol. Cell. Toxicol. 21 (2006).

[104] V. Andresen, S. Alexander, W. M. Heupel et al., Current Opin. Biotech. 2054 (2009).

[105] xfel.desy.de

[106] L. M. Kiernan, E. T. Kennedy, J-P. Mosnier et al., Phys. Rev. Lett. 722359 (1994).

[107] L. M. Kiernan, M. K. Lee, B. F. Sonntag, et al., J. Phys. B: At. Mol. Opt. Phys. 28 L161 (1995).

[108] S. H. Southworth, E. P. Kanter, B. Krassig et al., Phys. Rev. A 67062712 (2003)

[109] G. Zhu, M. Schuricke, J. Steinmann et al., PRL 103103008 (2009).

[110] H. N. Chapman, S. P. Hau-Riege, M. J. Bogan et al., Nature 448676 (2007).

[111] G. Lambert, T. Hara, D. Garzella et al., Nat. Phys. 4296 (2008).

[112] J. T. Costello, Nat. Photon. 267 (2008).

[113] http://sflash.desy.de

[114] B. Dromey, M. Zepf, A. Gopal A et al., Nat. Phys. 2456 (2006).

[115] B. Dromey, S. Kar, C. Bellei et al., Phys. Rev. Letts. 99085001 (2007). 


\section{Figure Captions.}

Figure 1: Cut through the electron-ion momentum spectrometer.

Figure 2: Schematic diagram of the experimental assignment.

Figure 3: Ion TOF spectra of Ar and $\mathrm{Kr}$ irradiated by $51 \mathrm{~nm}$ FEL pulses.

Figure 4: FEL power dependence of the ion yields for the individual charge states $\mathrm{Ar}^{n+}$

Figure 5: Photoion-photoion coincidence map of $\mathrm{N}_{2}$ irradiated by $24 \mathrm{eV}$ FEL pulses.

Figure 6: Photoion-photoion-photoion coincidence map for $\mathrm{C}^{2+}-\mathrm{O}^{2+}-\mathrm{O}^{2+}$ (and $\mathrm{C}^{+}-\mathrm{O}^{2+}{ }_{-}$ $\mathrm{O}^{2+}$ ) coincidences from $\mathrm{CO}_{2}$ irradiated by 62-nm EUV-FEL.

Figure 7: Kinetic energy release (KER) distributions for ion-pair formations from $\mathrm{N}_{2}$ irradiated by $24 \mathrm{eV}$ FEL pulses.

Figure 8: Ion time-of-flight mass/charge spectra of xenon taken at the photon energy of $93 \mathrm{eV}$ and different pulse irradiance levels [39].

Figure 9: Two-photon double ionisation cross-section of He as measured at FLASH [47] and using a HHG source $[49,50]$. Some theoretical predictions are shown for

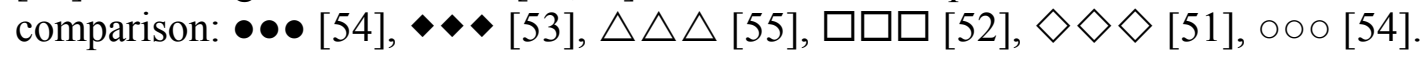

Figure 10: Ion momentum distributions for two-photon double ionization of $\mathrm{He}$ (a) and $\mathrm{Ne}(\mathrm{b})$ at $44 \mathrm{eV}$ integrated over the third (y-) direction. The laser polarization is along the $\mathrm{x}$-axis.

Figure 11: Kinetic energy release spectrum for TDPI of $\mathrm{D}_{2}$ (a) for $38 \mathrm{eV}$ at an estimated intensity of $10^{14} \mathrm{~W} / \mathrm{cm}^{2}$. Lines: theoretical results for sequential and direct TPDI (approximated) (see [66] for more details). (b) Asymmetry $\left(\beta_{2^{-}}\right)$parameter extracted from the coincident $\mathrm{D}^{+}$fragment angular distribution w.r.t. to the polarization axis (line: theoretical result [66]).

Figure 12: Kinetic energy of $\mathrm{N}^{2+}$ fragment ions emerging highly ionized $\mathrm{N}_{2}$ molecules as function of pump-probe delay time. Both pulses have identical intensities in the order of $10^{14} \mathrm{~W} / \mathrm{cm}^{2}$ (photon energy $38 \mathrm{eV}$ ).

Figure 13: (a) Schematic representation of electron dressing and concomitant sideband generation. (b) Photoelectron spectrum in the region of $\mathrm{He}^{-1} s^{-1}$ for overlapping FEL+IR beams showing the high and low-energy sidebands (SB). Dashed line: theoretical photoelectron spectrum of He obtained from TDSE calculations $[71,72]$. 
Figure 14: (a) Photoelectron spectra showing the He 1s photoelectron line and the high energy sidebands for high optical dressing fields $\left(\sim 6 \times 10^{11} \mathrm{~W} \mathrm{~cm}^{-2}\right)$ upon photoionization at $90.5 \mathrm{eV}$. Spectra are presented for different relative orientations between the linear polarization vectors of the FEL $(13.7 \mathrm{~nm})$ and IR $(800 \mathrm{~nm})$ laser fields. (b) Variation of the upper sideband yield in the low field regime $\left(\sim 8 \times 10^{10} \mathrm{~W}\right.$ $\mathrm{cm}^{-2}$ ) as a function of the relative angle between the linear polarization vectors of the FEL and IR laser fields. The solid line denotes the fit to the experimental data (circles). The results of time-dependent second-order perturbation theory (dashed line) and soft-photon approximation (dotted line) are almost identical [73].

Figure 15: Sidebands to high order on the Xe $5 \mathrm{p}^{-1}$ photoelectron lines at high IR (800 $\mathrm{nm})$ dressing field intensity.

Figure 16: Photoelectron spectrum of excited atomic hydrogen fragments in EUV dissociation (pump) - IR probe (ionization) experiments at FLASH.

Figure 17: (a) Velocity map imaging apparatus designed and built for the beamtime at FLASH. The FEL beam is sent into the re-focusing chamber where it is back-reflected by a flat mirror onto a spherical mirror, used closed to normal incidence, which focuses the light into the center of the interaction region of the spectrometer. The pump-probe laser enters the setup through a separate beam tube. On the optical breadboard, the $800 \mathrm{~nm}$ light is first frequency doubled, and the frequency-doubled pulse is delayed with respect to the fundamental before they are both focused by a lens into the spectrometer, where it crosses the FEL beam in the focus under a 10 degree angle. As the fundamental and its frequency-doubled replica are sent through different paths, the polarization of both beams can be chosen independently. The refocusing optics for the FEL are mounted in motorized mounts, providing precise control of the alignment also when the apparatus is under vacuum. Panel (b) shows the "spatial imaging mode" of VMI allowing us to observe the beam propagation in the detector plan. Panels (c-f) show the evolution of $\mathrm{H}^{+}$fragments as a function of the pump-probe time delay. When the EUV pulse arrives first, a new contribution is observed due to the very well known bond-softening effect.

Figure 18: Time evolution of $\mathrm{O}^{+}$ions resulting from dissociative ionization of $\mathrm{CO}_{2}{ }^{2+}$ by a $46 \mathrm{eV}$ FEL pulse. In panel (a), the FEL pulse precedes the IR pulse. The resulting fragments through dissociation (central ring) or Coulomb explosion (outer two rings) have an isotropic angular distribution. Panel (b) shows that at overlap between the two pulses, the angular distribution of $\mathrm{O}^{+}$ions peaks along the laser polarization, which indicates an alignment of the molecules. This situation is periodically repeated after the pulse has ended, as indicated in panel (c) that display the time evolution of $<\cos ^{2} \theta>$ as a function of the delay between the IR pulse and the EUV probe pulse (see text). Reproduced from reference 92.

Figure 19: $\mathrm{Br}^{2+}$ ions resulting from dissociation of $\mathrm{Br}_{2}$ by $13 \mathrm{~nm}$ light from FLASH. Panels (a), (b) and (c) show momentum distributions. In panel (a), the FEL pulse alone is impinging on the molecules, resulting in fragmentation through dissociation (central ring) or Coulomb explosion (outer two rings). In panel (b), the FEL pulse is preceded by a $400 \mathrm{~nm}$ pump pulse which induces dissociation of the neutral molecule, resulting in a sharp central ring representing fragments with a well defined energy gained in the dissociation channel. In panel (c), an IR alignment pulse is sent in 
around $1 \mathrm{ps}$ before the other two pulses, exciting a coherent superposition of rotational states in the molecules, causing them to impulsively align after the IR pulse is over, resulting in a momentum distribution which is peaked along the laser polarization axis. Panel (d) shows the kinetic energy distributions as a function of delay between the $400 \mathrm{~nm}$ dissociation pulse and the FEL pulse. A sharp timedependent contribution is observed due to an additional kinetic energy from Coulomb repulsion being imparted to the dissociating atoms when they are ionized at short inter-nuclear distances.

Figure 20: Photoelectron momentum distributions from $\mathrm{Br}_{2}$ ionized by $13 \mathrm{~nm}$ light from FLASH. Panel (a) shows data acquired without apertures along the FEL path resulting in a large background from scattered EUV. Panel (b) shows an image acquired under the same conditions but with apertures installed in the apparatus with the purpose of blocking scattered EUV photons and preventing them from hitting the detector. The improvement is clearly recognized, and in panel (c) the corresponding photoelectron energy spectrum is shown, revealing a multitude of peaks resulting from ionization of $\mathrm{Br}_{2}$ as well as of helium which was used as a carrier gas.

Figure 21: Ion TOF spectrum for different X-ray beam intensities. The X-ray photon energy is $1 \mathrm{keV}$ and the pulse width is $280 \mathrm{fs}$. The ion yields are normalized using the $\mathrm{N}^{2+}$ signal. 


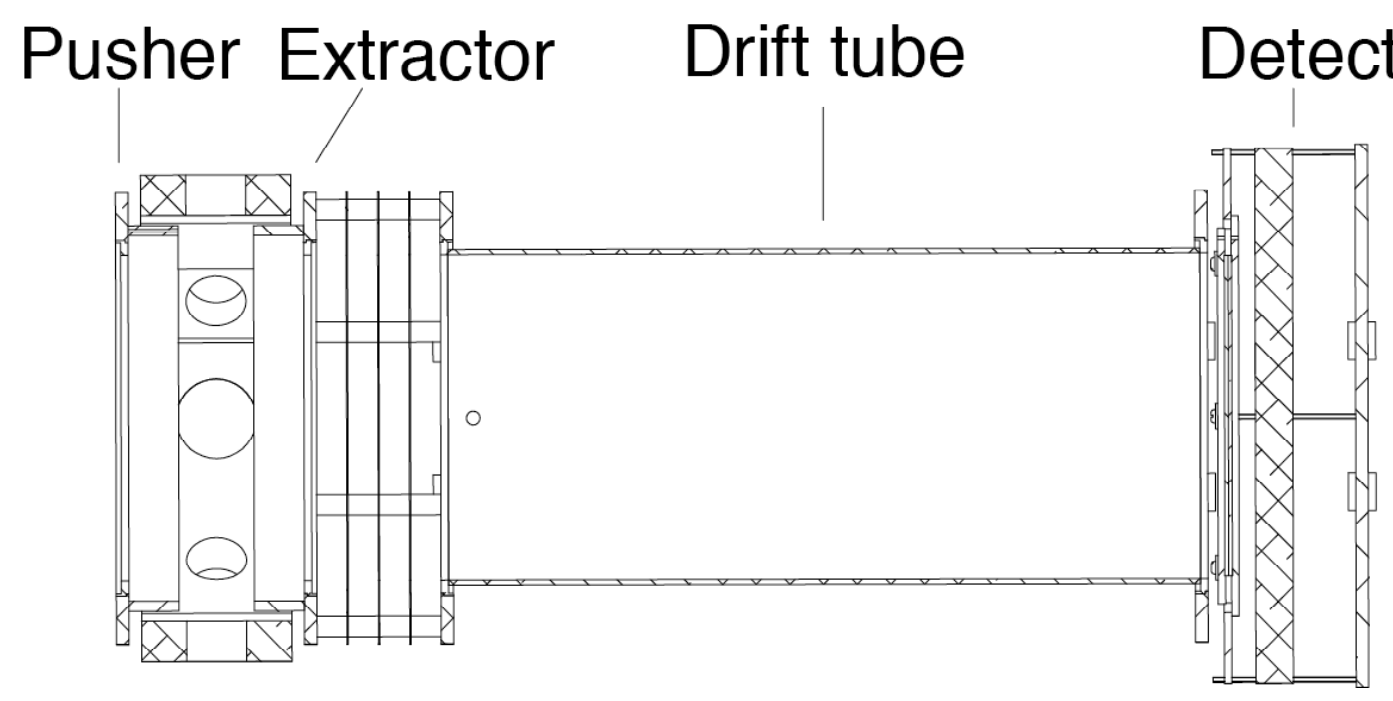




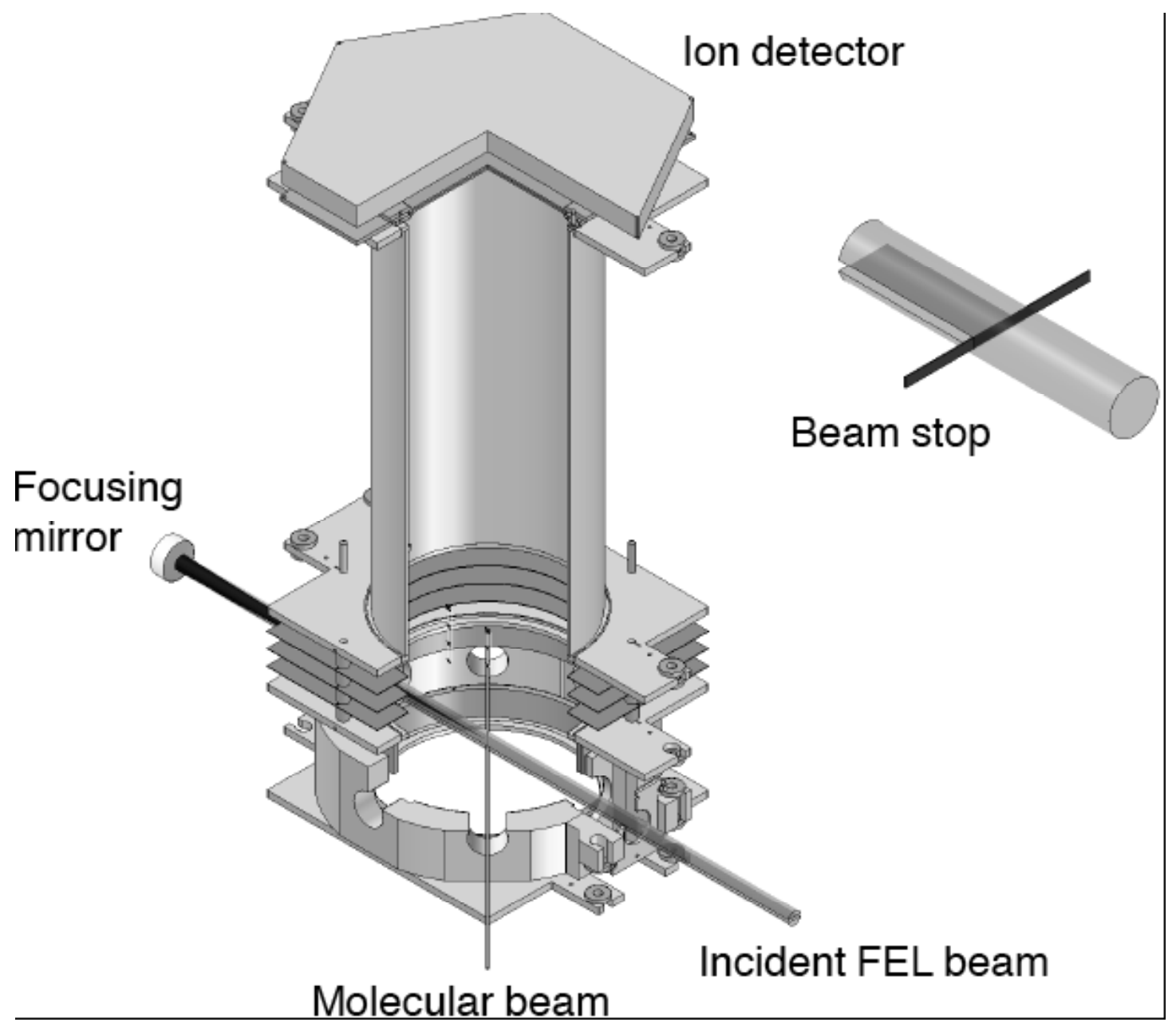




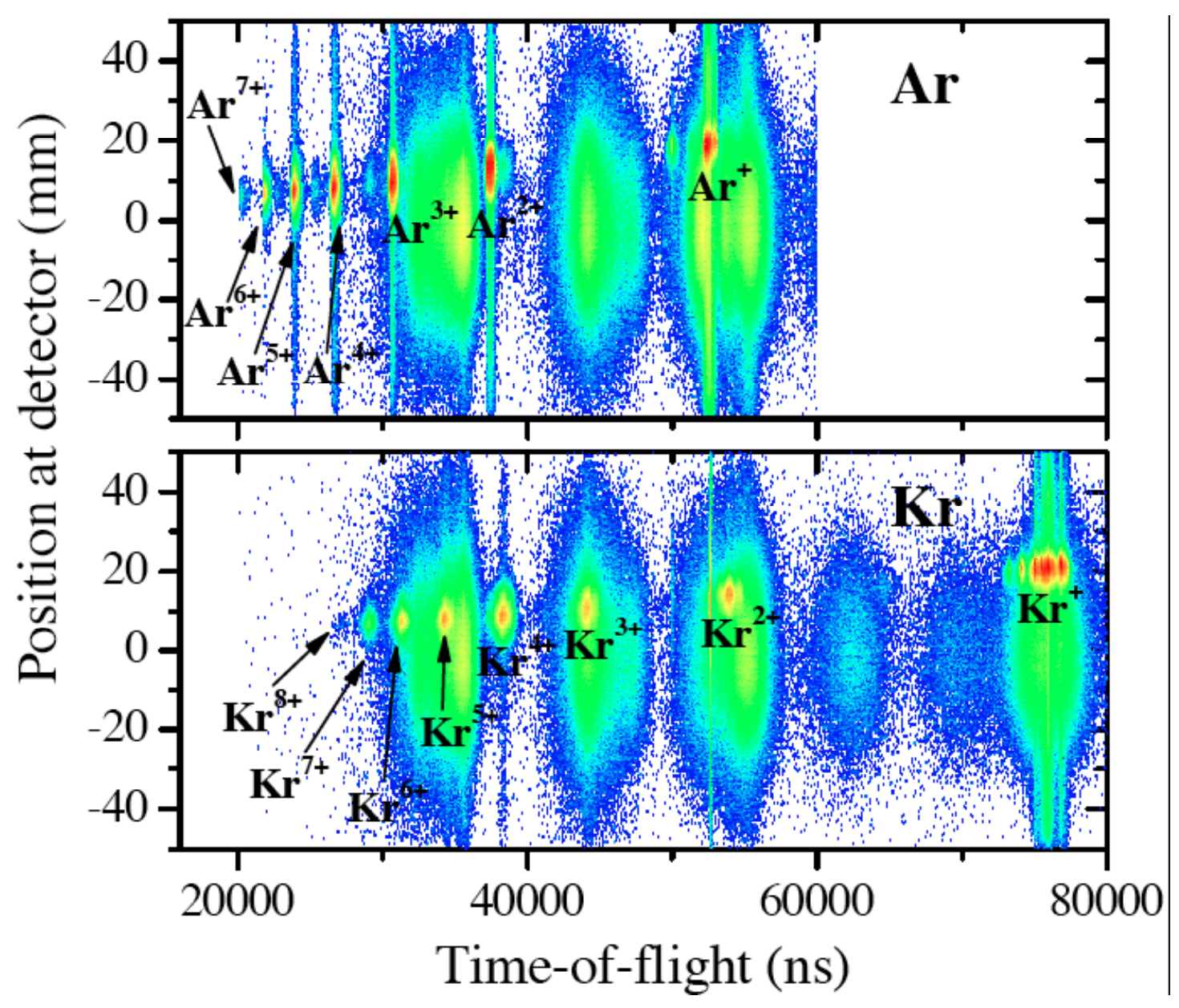




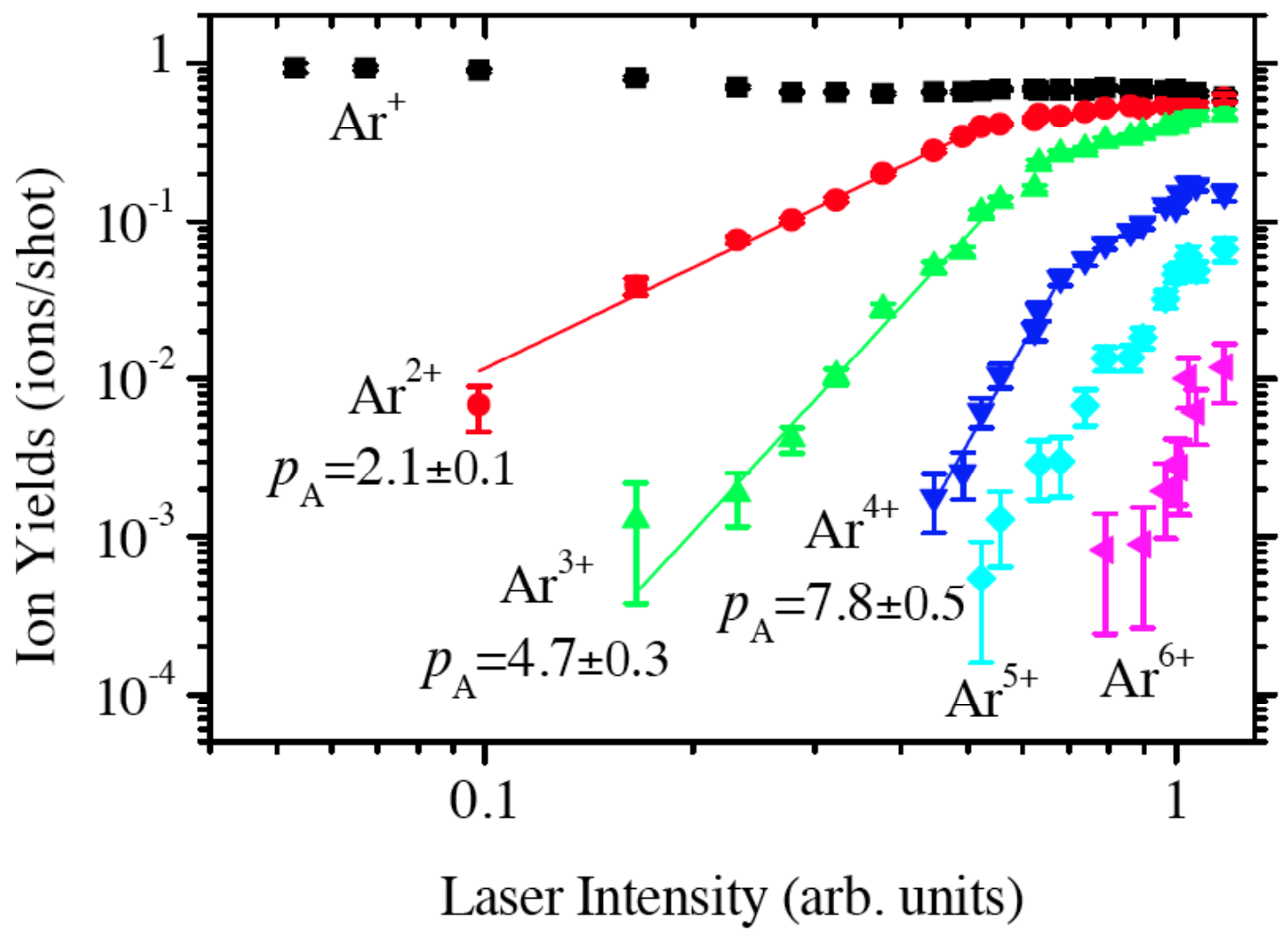




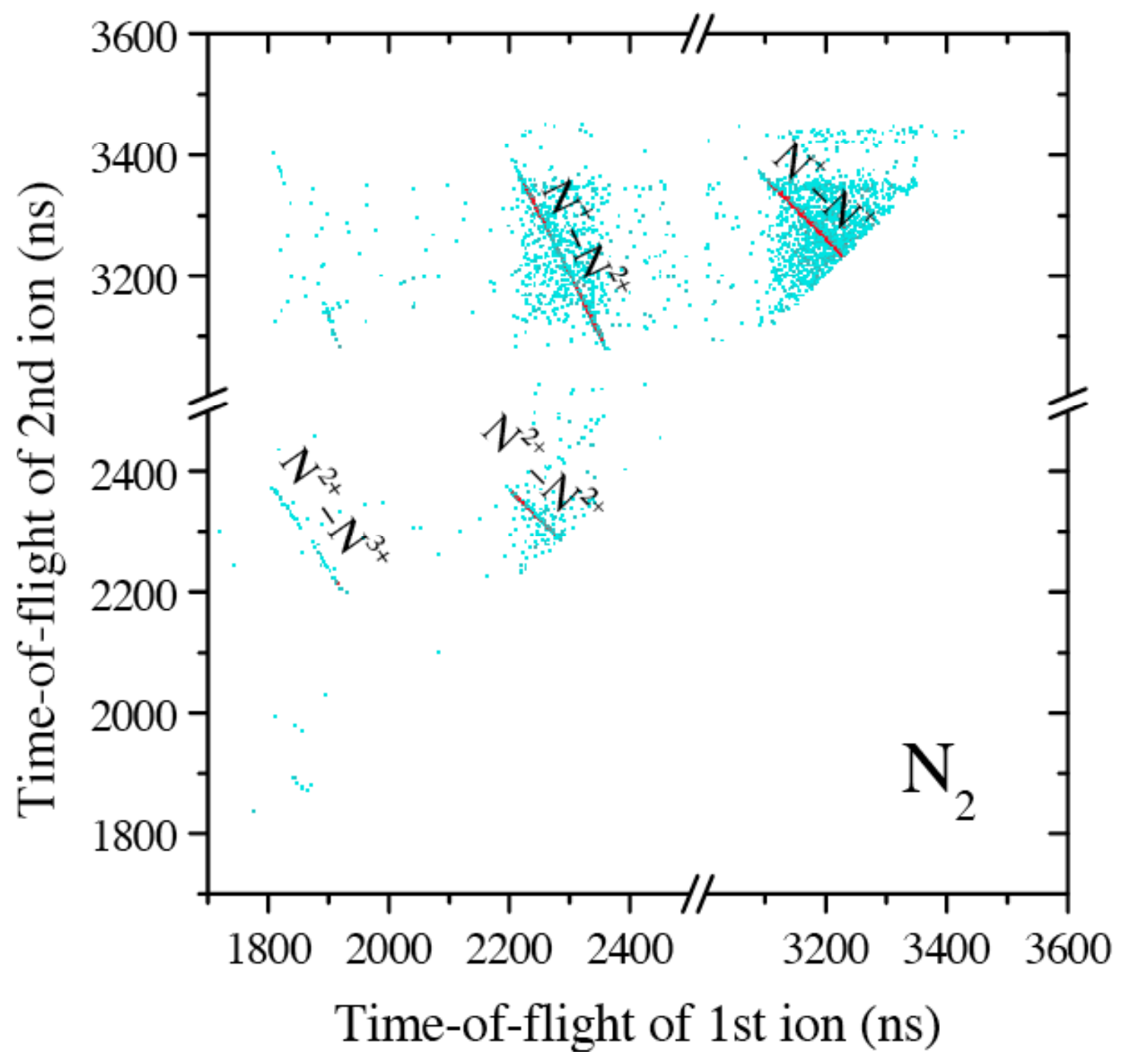




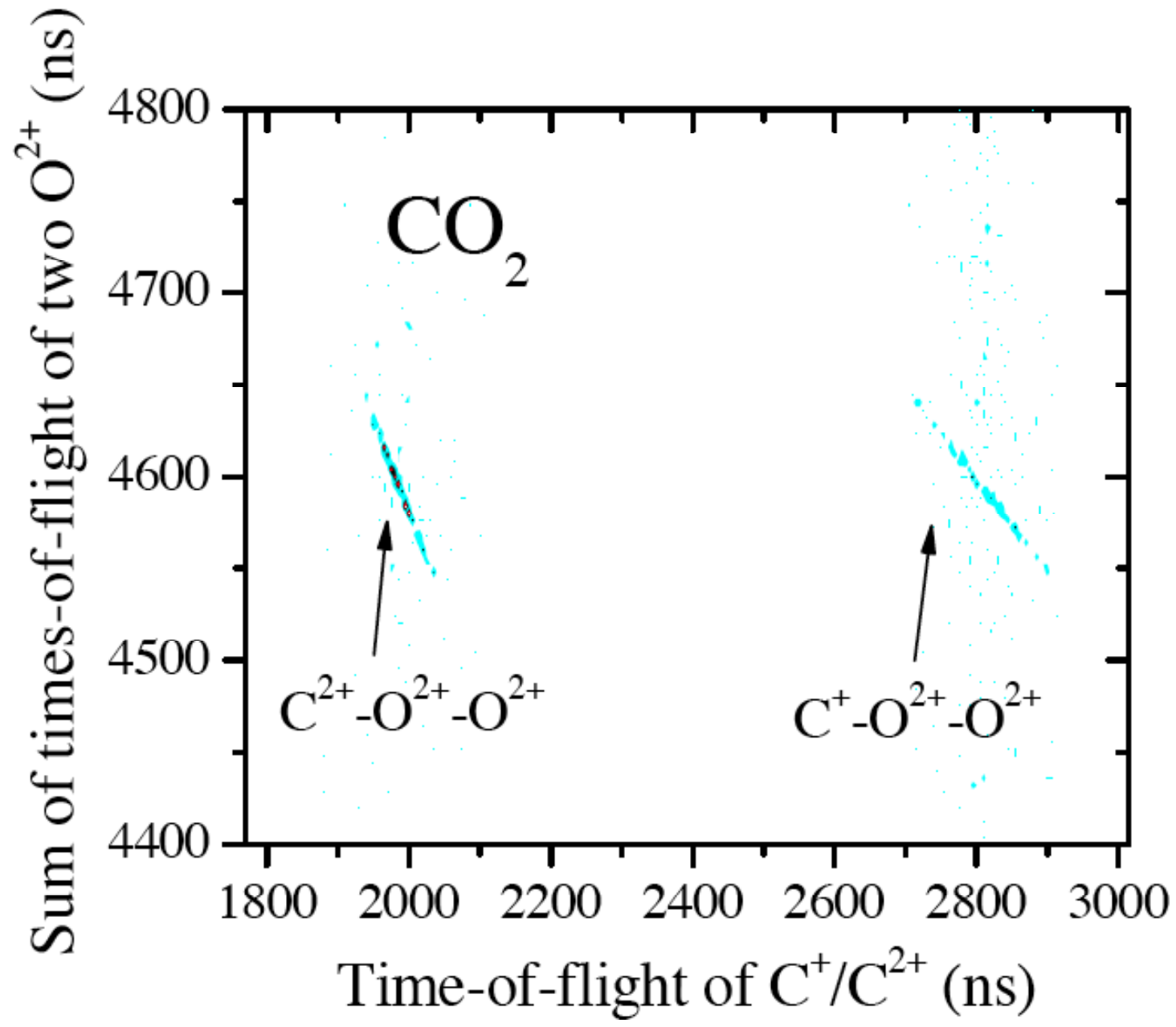




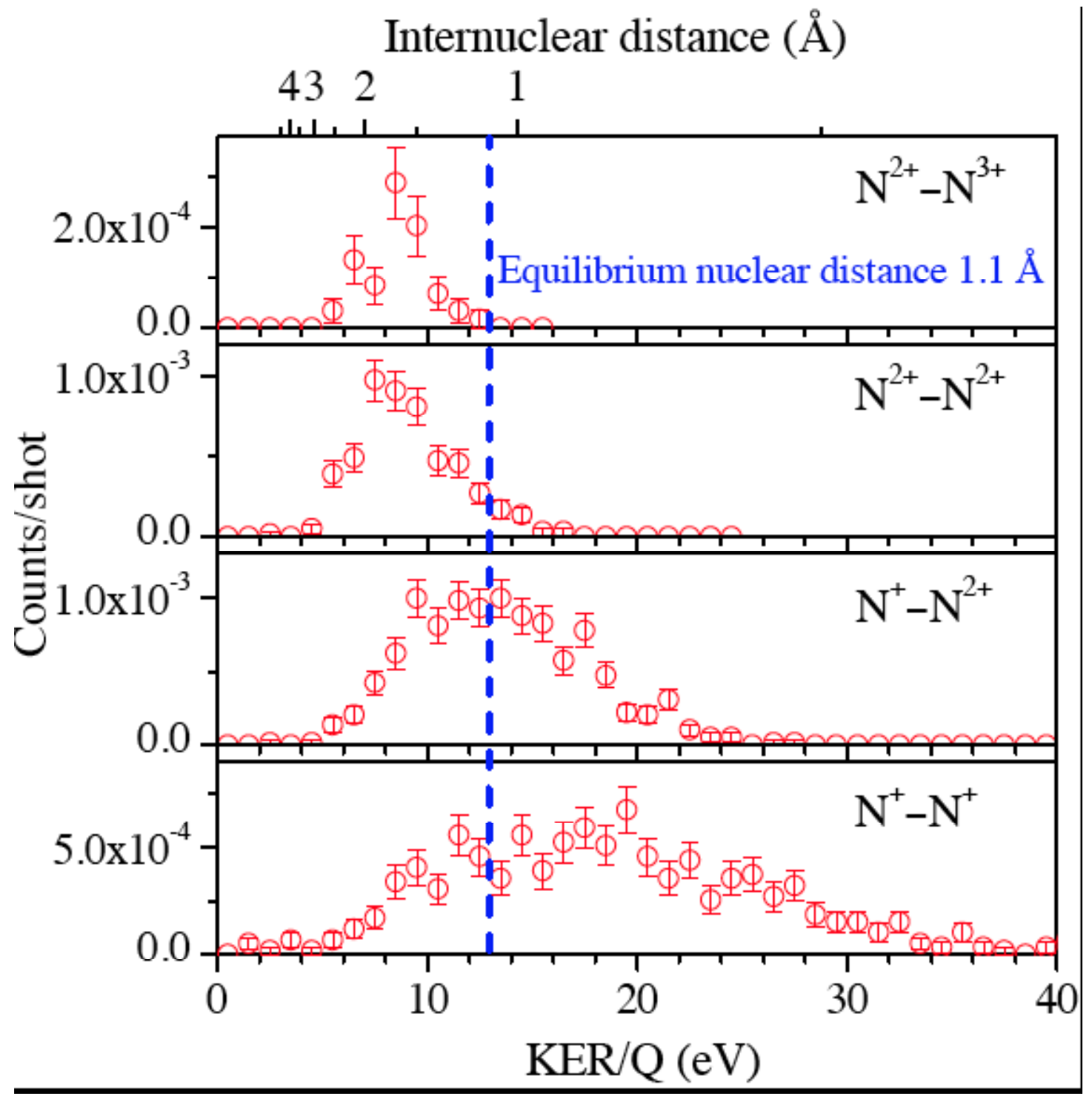




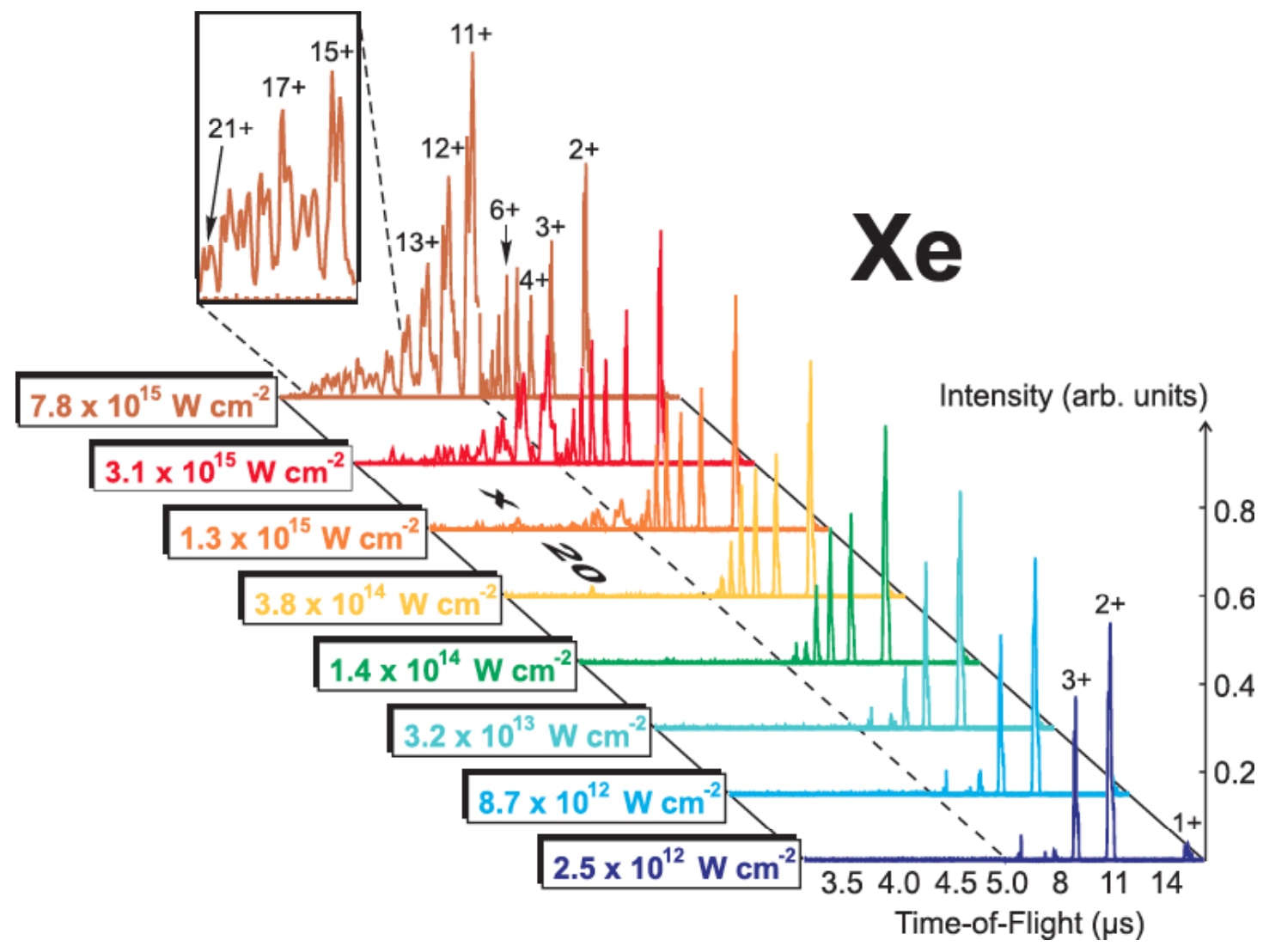




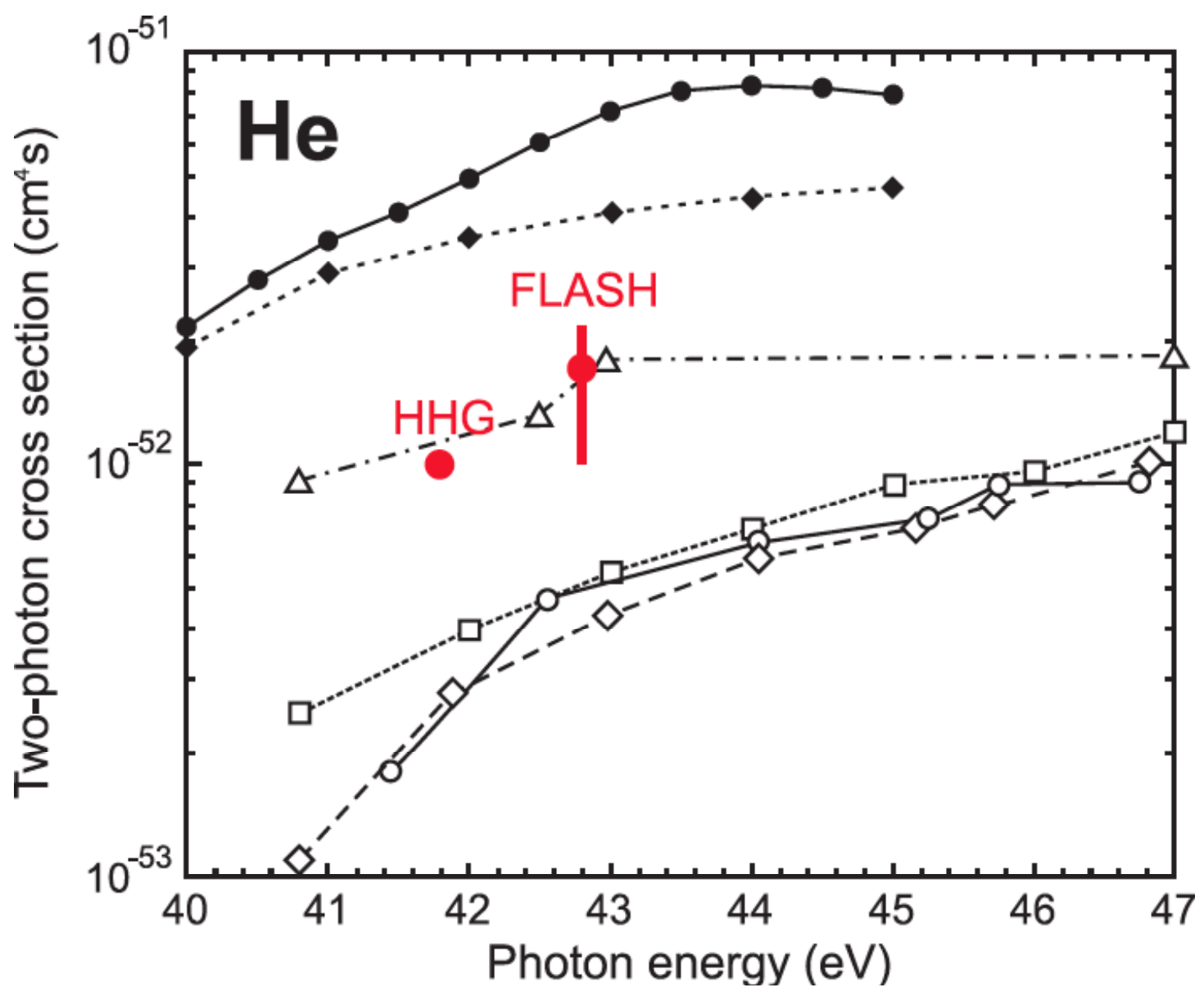




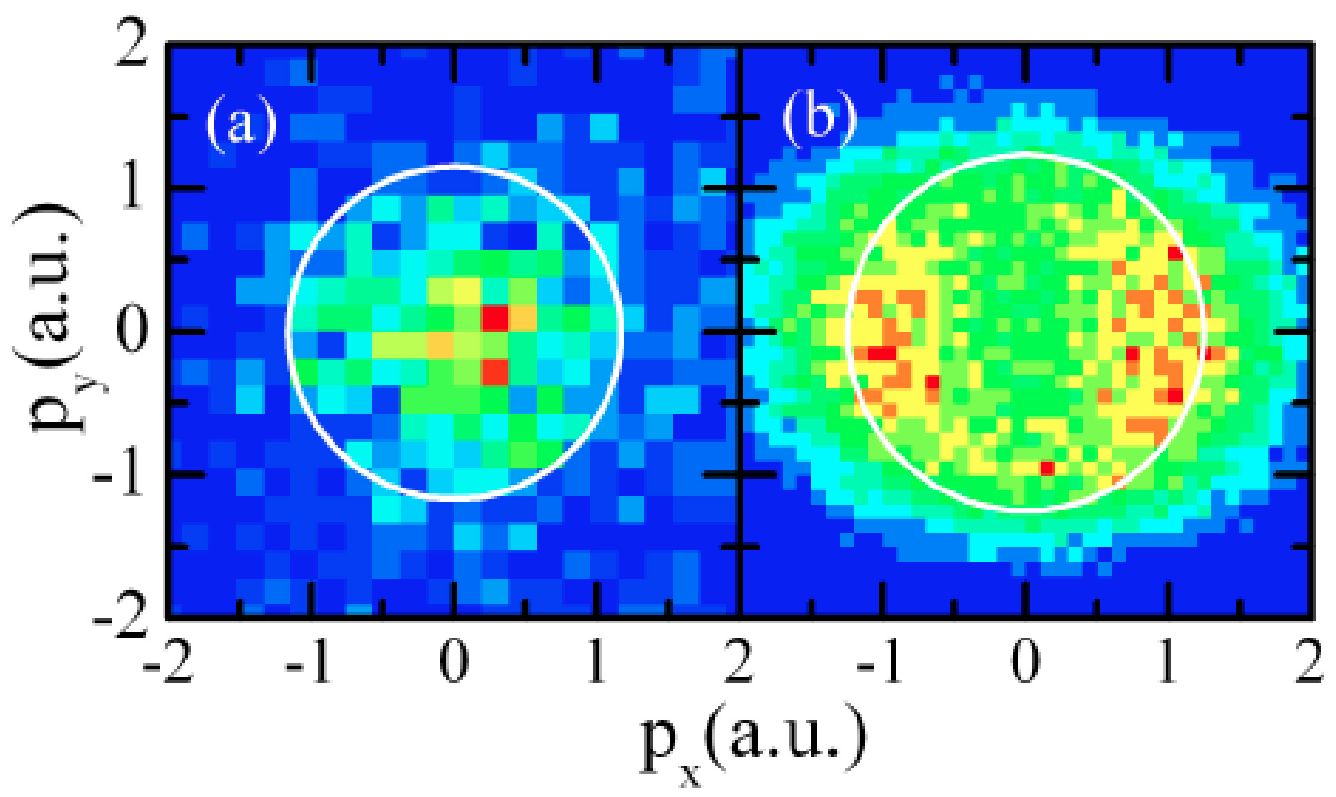




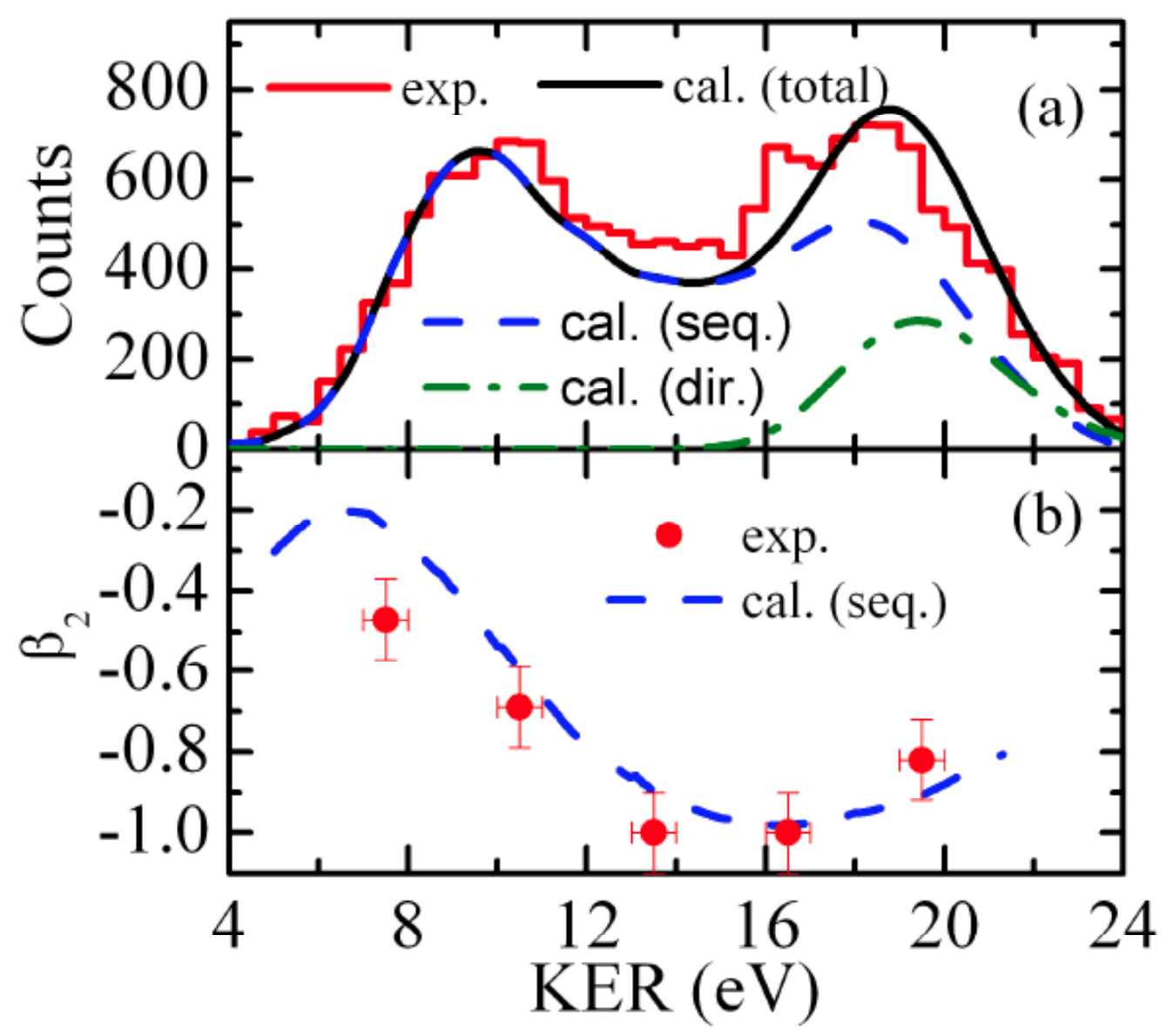




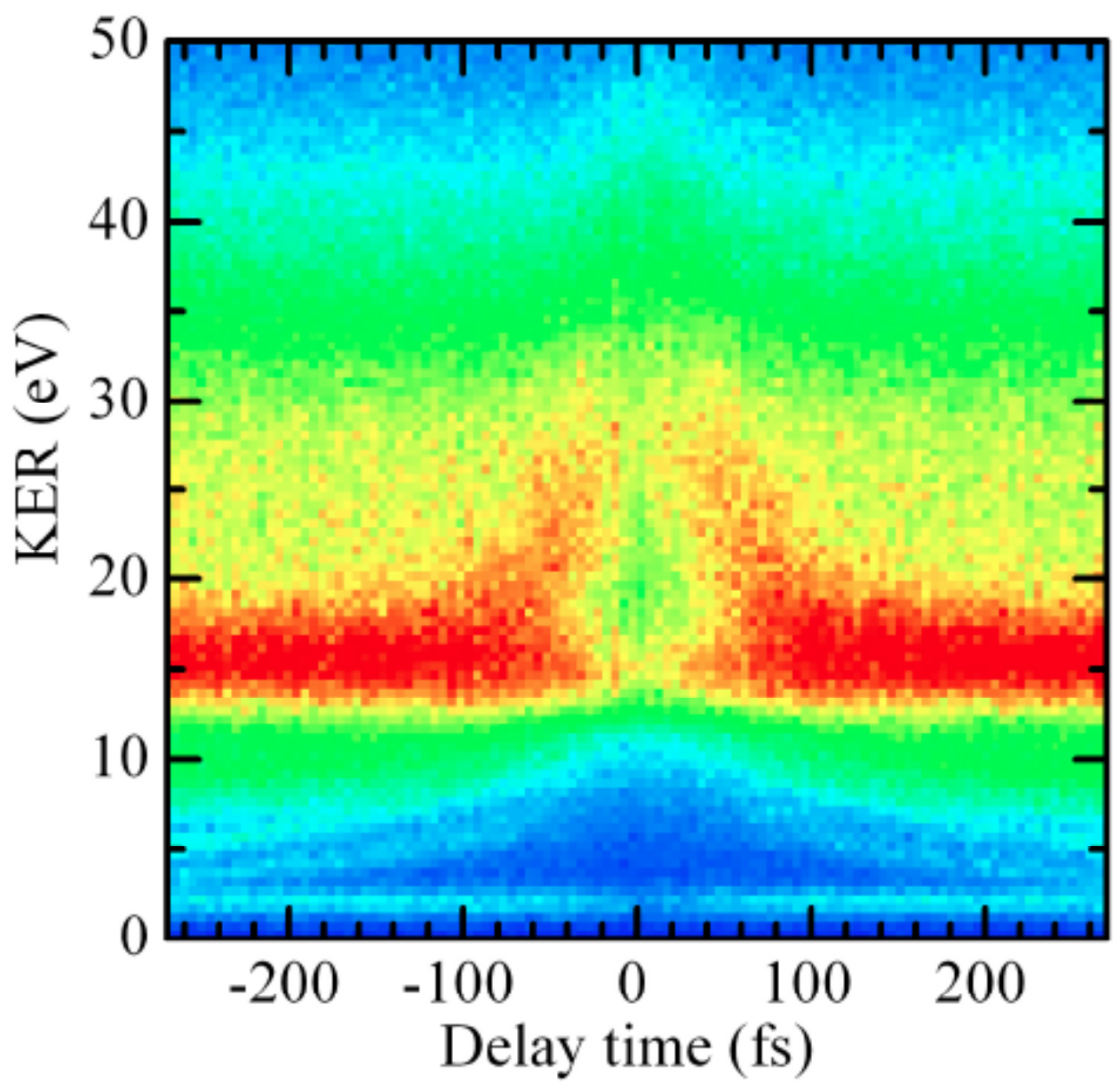





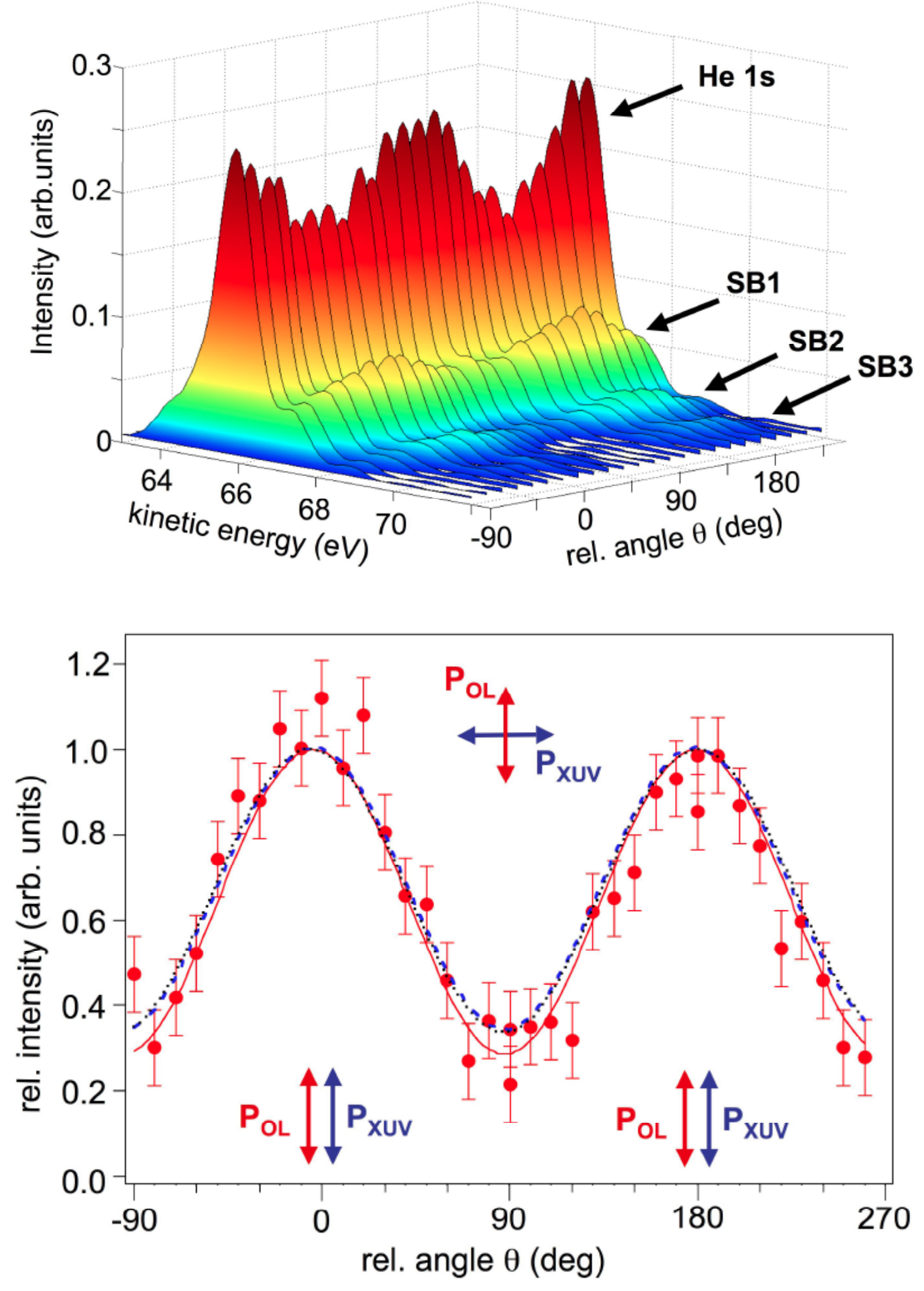


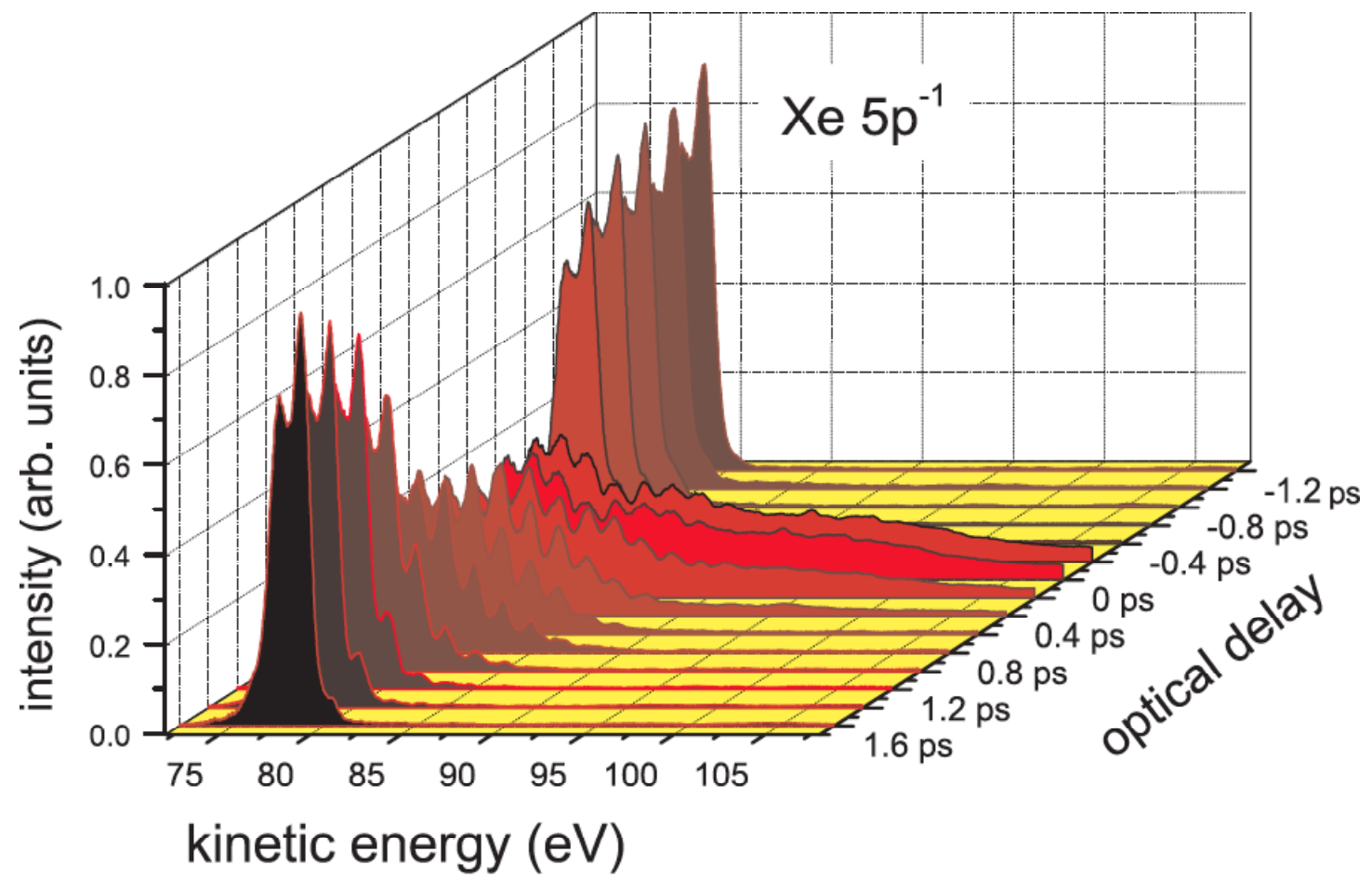




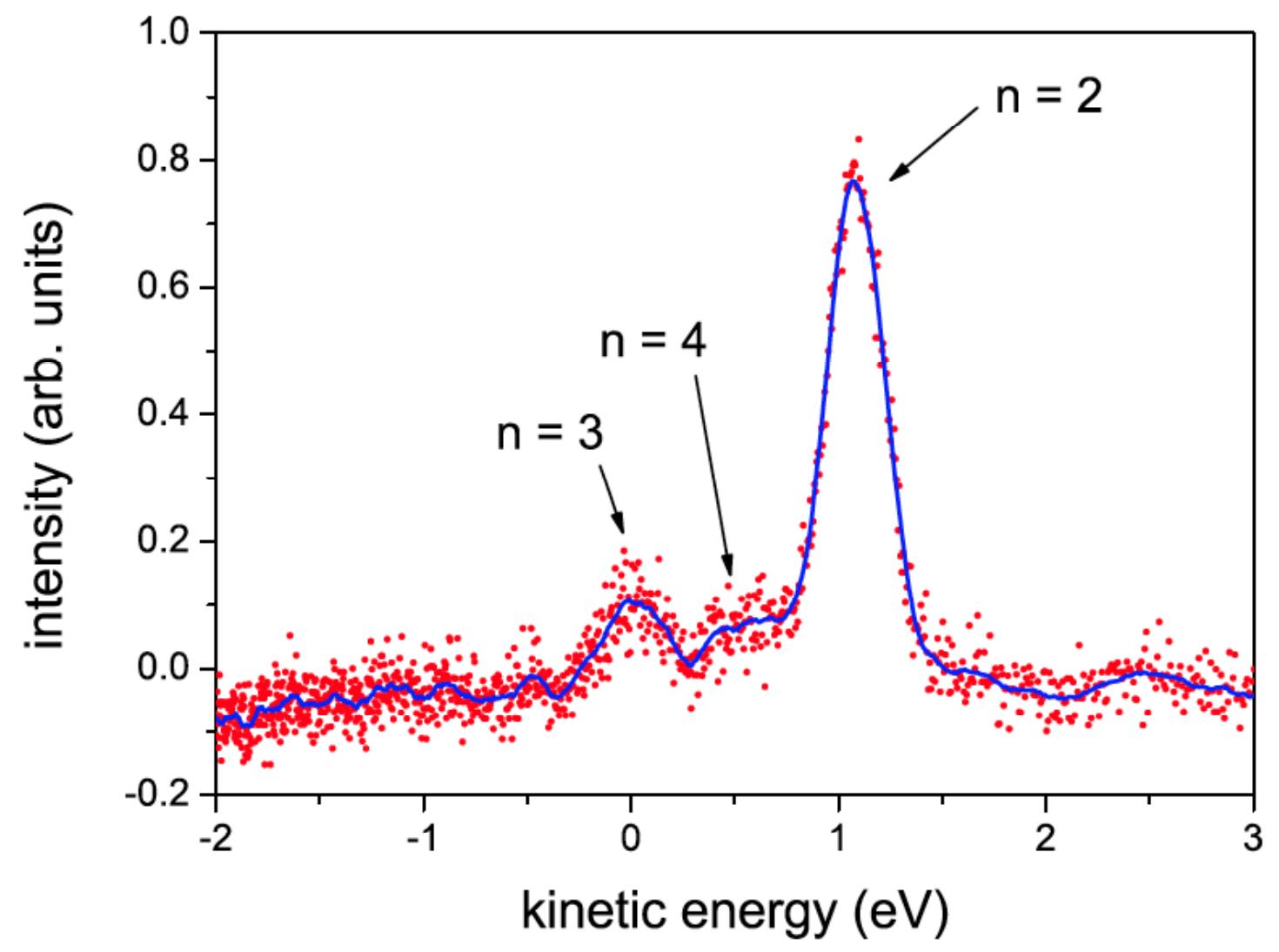




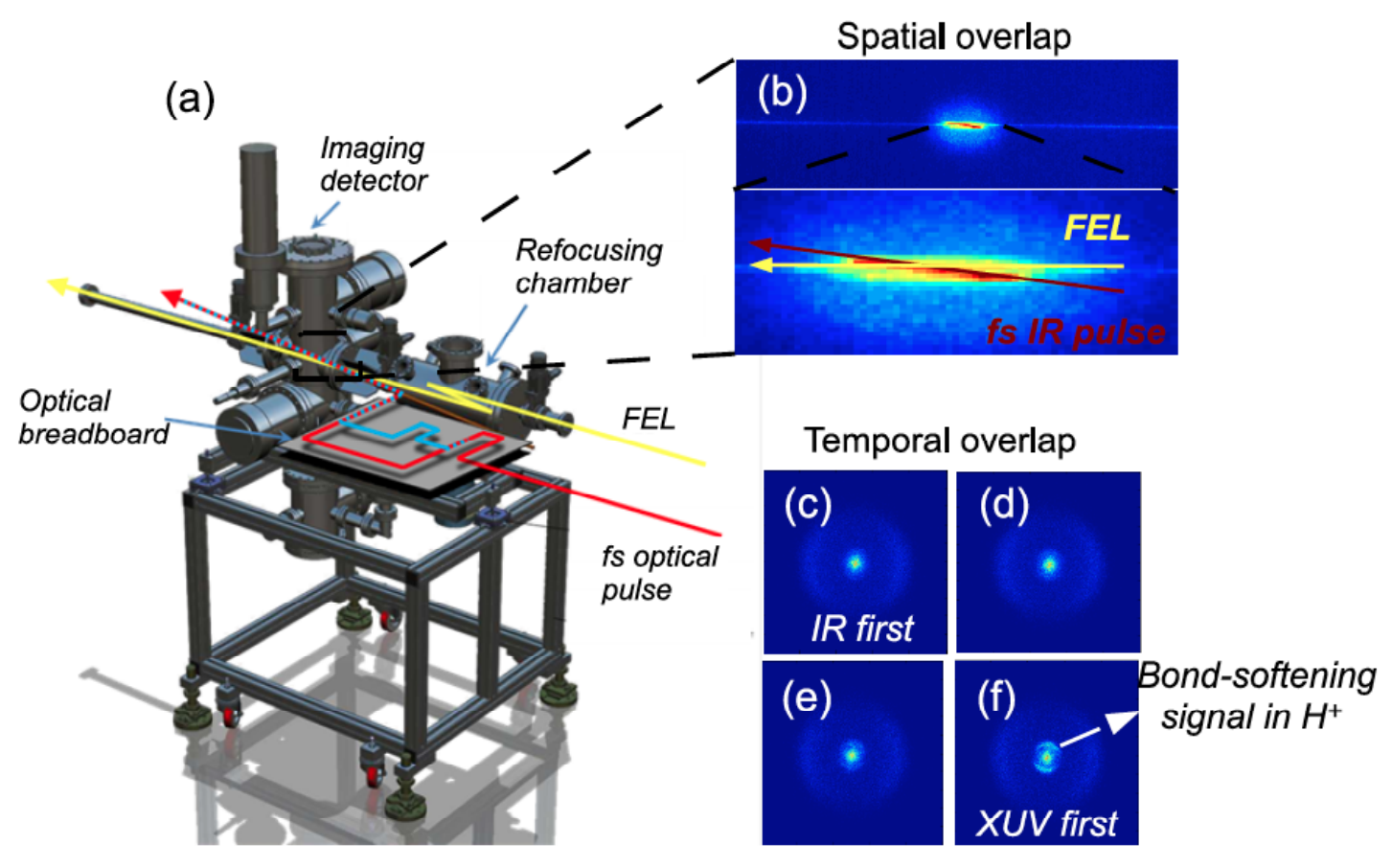



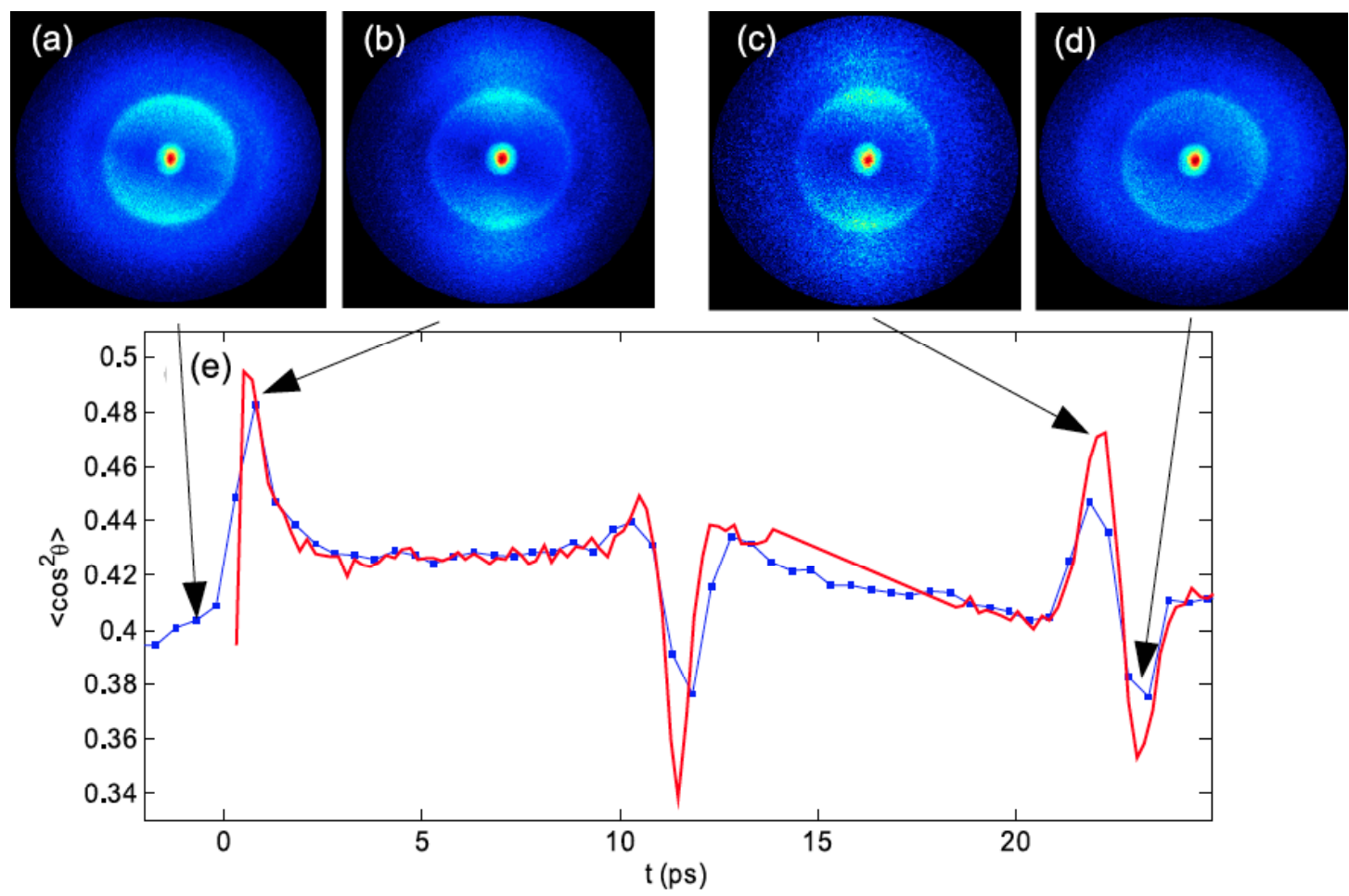


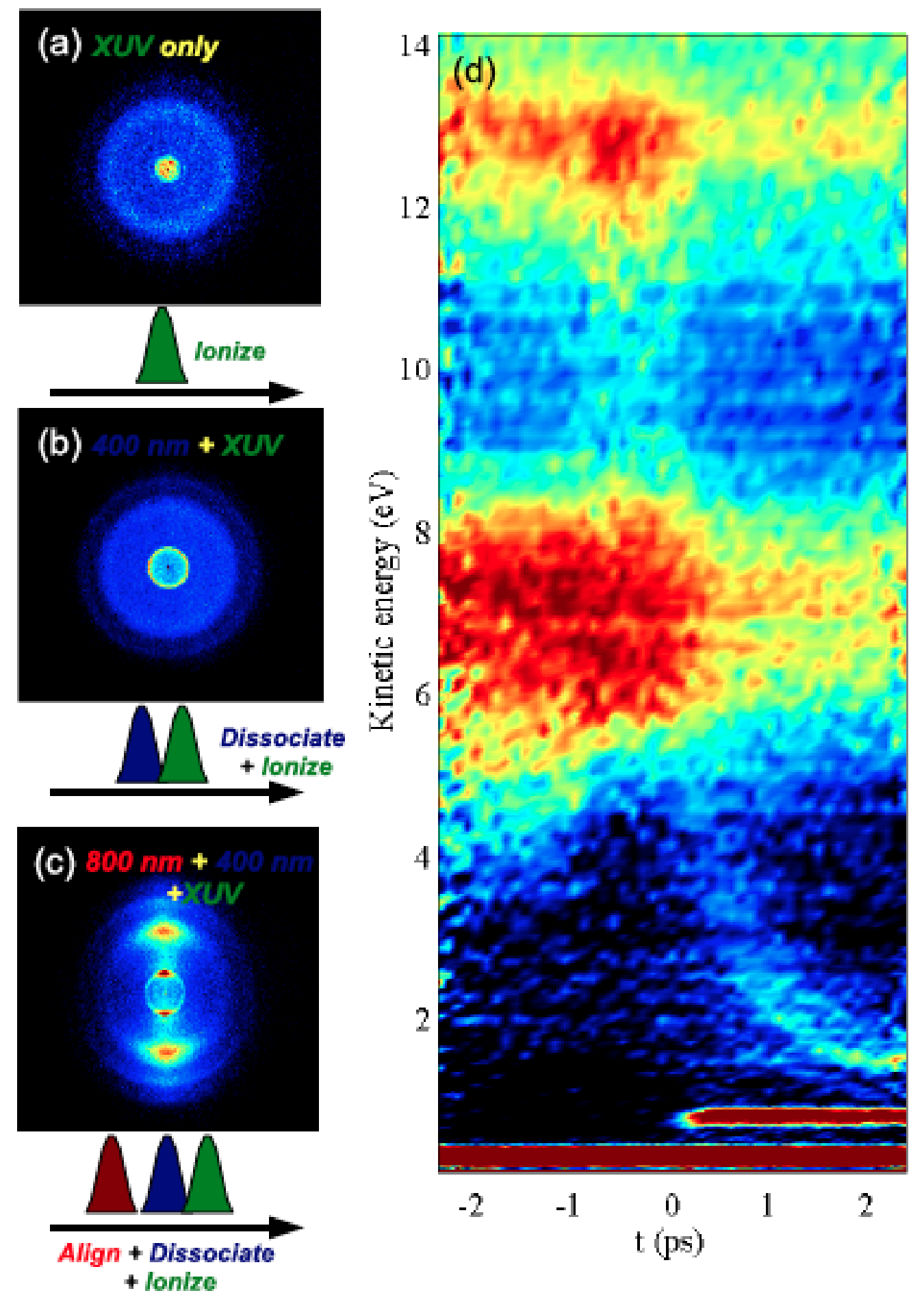



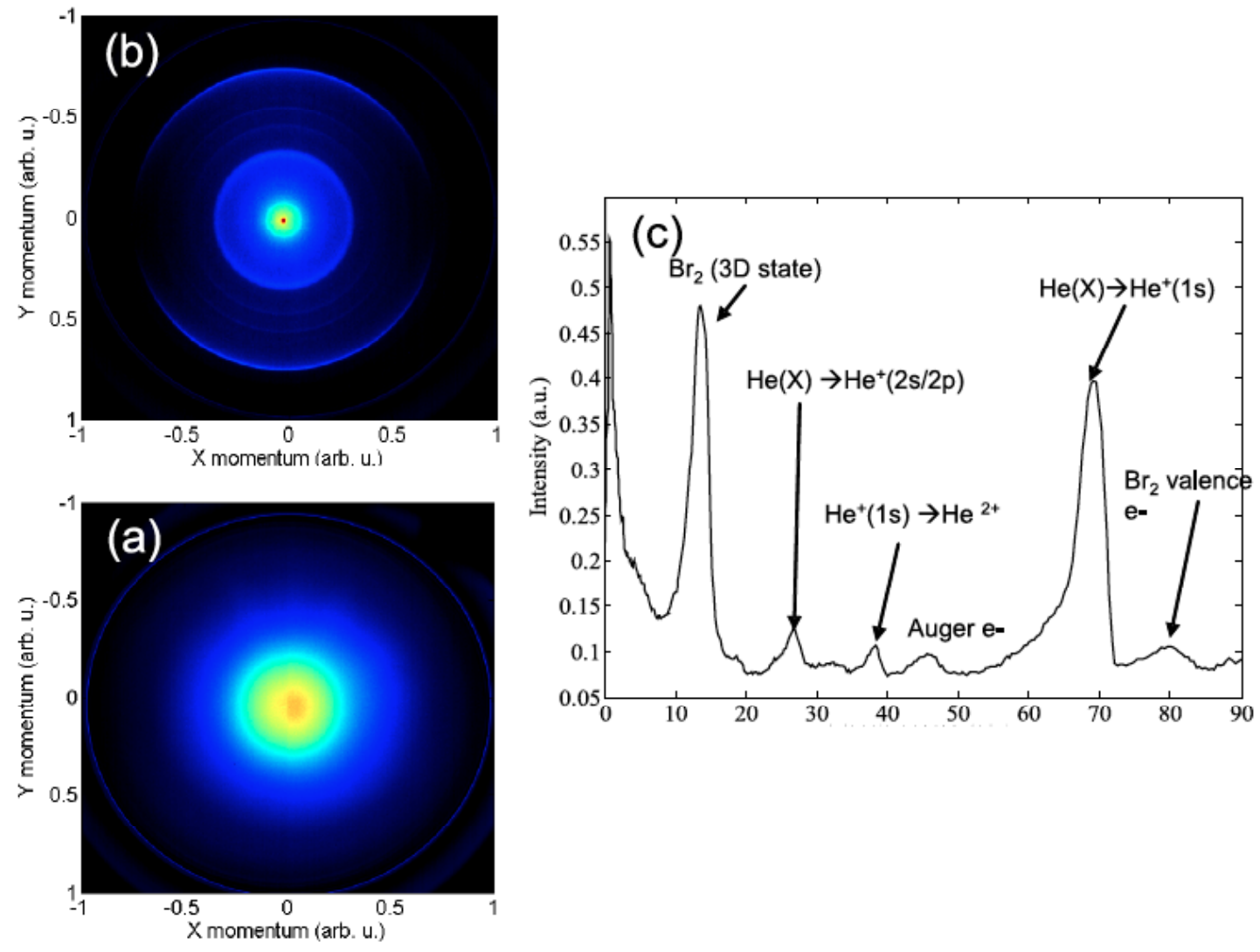


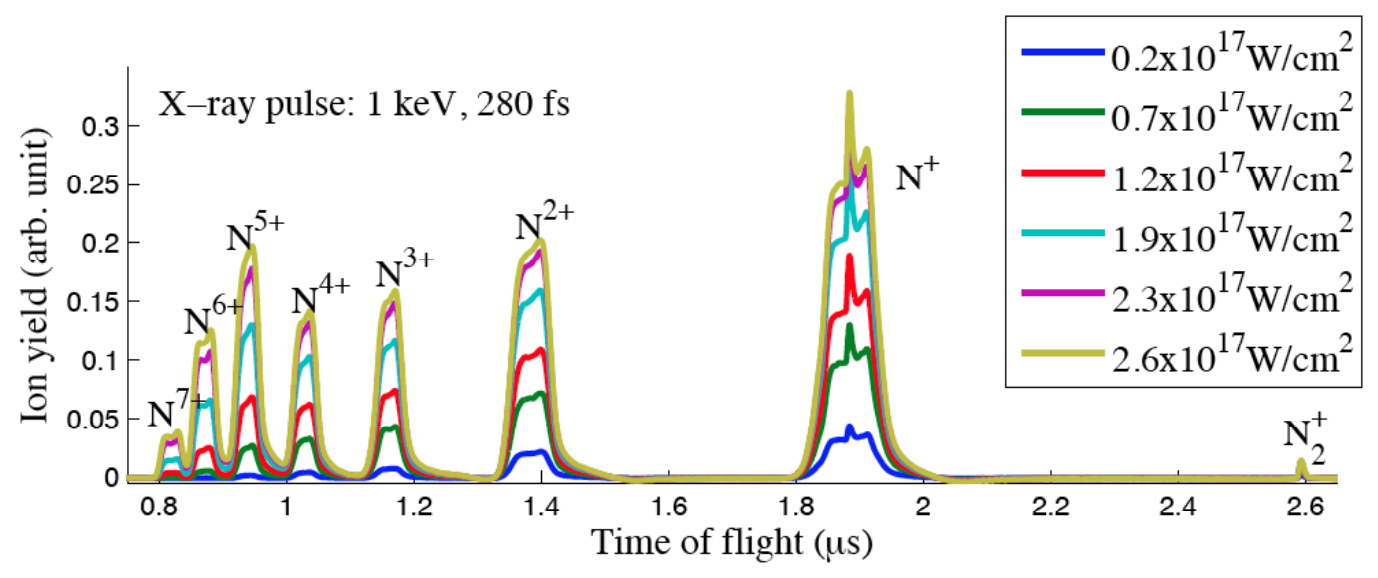

